\title{
Article \\ Effects of Bulk Flow Pulsations on Film Cooling with Two Sister Holes
}

\author{
Seung Il Baek and Joon Ahn *
}

check for

Citation: Baek, S.I.; Ahn, J. Effects of Bulk Flow Pulsations on Film Cooling with Two Sister Holes. Appl. Sci. 2021, 11, 1537. https://doi.org/10.3390/ app11041537

Academic Editor: Francesca Scargiali Received: 31 December 2020

Accepted: 3 February 2021

Published: 8 February 2021

Publisher's Note: MDPI stays neutral with regard to jurisdictional claims in published maps and institutional affiliations.

Copyright: (c) 2021 by the authors. Licensee MDPI, Basel, Switzerland. This article is an open access article distributed under the terms and conditions of the Creative Commons Attribution (CC BY) license (https:// creativecommons.org/licenses/by/ $4.0 /)$.
School of Mechanical Engineering, Kookmin University, Seoul 02707, Korea; greenjet50@gmail.com

* Correspondence: jahn@kookmin.ac.kr; Tel.: +82-2-910-4833

\begin{abstract}
In a triple-hole system comprising a primary hole and two sister holes, when the sister holes are positioned slightly downstream of the main hole under steady flow conditions, their jets generate an anti-counter-rotating vortex pair. Vortex interactions between the jets increase the effectiveness of adiabatic film cooling. In this study, a series of large-eddy simulations were conducted to understand how pulsations in the main flow affect film cooling in a triple hole. To understand the effects of pulsations on film cooling performance is important for better cooling design of the gas turbine engines. The numerical simulations were carried out on a flat plate geometry with a triple cylindrical hole system at $35^{\circ}$ injection angle. The pulsations were approximately sinusoidal, and their effect on film cooling was investigated at several frequencies $(2,16$, and $32 \mathrm{~Hz})$ and Strouhal numbers $(\mathrm{Sr}=0.1005,0.8043$, and 1.6085$)$ at an average blowing ratio of 0.5 . The results for the triple-hole system were compared with those for a single hole for the same amount of cooling air and the same cross-sectional area of the holes. Increasing the Strouhal number of the main flow decreased $\eta$ in both systems. However, at each Strouhal number, $\eta$ was higher in the triple hole. Furthermore, the triple-hole system was found to be better for film cooling than a single-hole system for higher values of the pulsation Strouhal number. Contours of time-averaged film cooling effectiveness and dimensionless temperature, instantaneous film cooling effectiveness contours on a test plate, mean velocity magnitude contours in the hole, and Q-contours for the triple holes under the application of pulsations to the flow were investigated.
\end{abstract}

Keywords: large-eddy simulation; turbine blade cooling system; adiabatic film cooling effectiveness; bulk flow pulsation; triple cooling hole configuration

\section{Introduction}

The efficiency of the gas turbine can be improved by raising the inlet temperature [1]. However, the turbine blade temperature should remain below the acceptable limit [2]. Film cooling is a widely used in gas turbines [3]. The physics of film cooling under a steady main flow has been widely investigated. Especially, intended vortex interactions could be induced between cooling jets by using specific cooling hole arrangements instead of the single hole. Heidmann et al. [4] numerically showed that compared with a one-hole system, the use of three holes (a primary hole and two side holes) increased the film cooling effectiveness at $M=1.0$. They also reported that manufacturing triple cylindrical holes was more economical than manufacturing holes with other shapes. Using square sister holes, Javadi et al. [5] controlled the vortex interactions between injectant and main flow at the blowing ratio, $M$ of 0.5 . They numerically found that two sister holes reduced the mixing between the coolant injectant and the crossflow, thereby increasing $\eta$. Ely et al. [6] numerically investigated the increase in $\eta$ after forming two sister holes [3]. They varied the blowing ratio among the values $0.2,0.5,1.0$, and 1.5 and reported an obvious improvement in $\eta$ at high $M$. Choi et al. [7] numerically investigated performance of the film cooling for double holes by changing design variables such as the lateral ejection angles and streamwise and lateral distances between the holes. They found that the lateral distance 
and lateral ejection angles significantly influenced the film cooling performance while the effect of the streamwise distance on the film cooling effectiveness was considerably less. Wu et al. [8] determined the cooling performance of three types of sister hole configurations by using experimental and numerical methods. They stated that the effectiveness of the film cooling was considerably higher for the triple-hole configurations compared with that for a single-hole configuration under steady flow conditions.

However, in real flow fields, the mainstream flow could be unsteady. It could be disturbed by many reasons such as potential flow interactions, and freestream turbulence generated in the combustor or passing wakes [9]. The effect of flow instabilities on the film cooling performance for the triple hole system has not been studied. Understanding the effect of main flow pulsation on the film cooling for the triple holes would help for making better cooling design of the gas turbine engines. Kim et al. [10] observed that most previous computational fluid dynamics (CFD) studies on film cooling had been conducted under steady flow conditions. Few studies have considered the effect of pulsations, caused by flow instabilities, in the main flow. Gau et al. [11] experimentally studied the effect of swirling flow in mainstream flow on $\eta$. They showed that if the swirling number, which represents the axial flux of the swirl momentum, is increased, $\eta$ decreases due to improved mixing between the main flow and cooling jet. Coulthard et al. [12] studied the film cooling effect of a pulsating injectant by using experimental method, and they reported that steady coolant injection maximized $\eta$ [2]. When the coolant injection was pulsed, $\eta$ reduced with increase of Strouhal number at $M=0.5$.

Nikitopoulos et al. [13] reported that coolant pulsation can control the film cooling performance. To understand the physics in the pulsed film-cooling flow field, researchers have investigated the optimal parameter range of coolant pulsation. Ekkad et al. [14] experimentally studied the effect of cooling jet pulsation on the film cooling in a turbine blade model. They found that jet propulsion weakened the jet liftoff at high blowing ratios, increasing the spread of the coolant on the wall. However, when the main flow velocity is disturbed by flow interactions, shock waves, turbulence generated in the combustion chamber, or passing shock waves on the turbine blade, the disturbance patterns are more similar to sinusoidal waves than pulses [9]. In an experimental study, Seo et al. [9] showed how sinusoidal mainstream pulsations influence film cooling. They reported that for a small $L / D$ ratio, increasing the pulsation frequency from 0 to $32 \mathrm{~Hz}$ at the blowing ratio, $M$ of 0.5 decreased the adiabatic film cooling effectiveness and increased the iso-energetic Stanton number ratio. Gao et al. [15] reported the effect of vane passage vortex generation on film cooling by using a pressure-sensitive paint. The strength of the vortex was adjusted by changing the attack angle of the main flow to the delta wing. They found that a stronger vortex resulted in increased the mixing, leading to a decreasing $\eta$.

Xiao et al. [16] showed a fractal solution to study the Kozeny-Carman constant and permeability for fibrous porous media. They stated that if the particle diameter was increased, the absolute permeability was increased and if the tortuosity fractal dimension was increased, the Kozeny-Carman constant was increased, however, the dimensionless permeability was decreased. Liang et al. [17] proposed that a fractal theory of porous media in order to quantify the porous media's effective electrolyte diffusivity. The analytical results showed a good match with the experimental data.

In this study, an analysis was performed using a large-eddy simulation (LES). Although a LES incurs higher computational cost than Reynolds-averaged Navier-Stokes (RANS), the RANS approach cannot accurately predict complex flow interactions because it involves ensemble-averaging the turbulent fluctuations in the flow. By contrast, the LES approach resolves large-scale turbulent eddies, enabling accurate predictions of complex flows [2,18]. Johnson et al. [19] found that an LES model showed considerably more spreading of the coolant jet compared with RANS turbulence models and that the LES results were similar to real film cooling fields. Baldauf et al. [20] found that at the low blowing ratios, the effectiveness of the film cooling decreased rapidly as the coolant travelled downstream, despite high film cooling effectiveness around the cooling hole. This can be attributed to the 
small mass flow rate of the coolant. Furthermore, low blowing ratios cause the detrimental ingestion of the hot mainstream gas into the hole [9,21]. Bogard [22] reported that the overall film cooling effectiveness increases with the blowing ratio up to the blowing ratio of 0.6 . He observed that when the blowing ratio exceeds 0.85 , the overall effectiveness decreases because of the separation of the coolant jet from the wall. Therefore, in this study, the average blowing ratio was fixed at 0.5 . A sinusoidal pulsation in the main flow causes the difference between the static pressures around the inlet and the exit of the hole to oscillate, which in turn makes the coolant jet oscillate. Therefore, in the current study, a sinusoidal waveform was simultaneously applied to the main flow inlet and the plenum inlet with the same frequency.

By performing an LES, the current study investigated the effect of oscillating the main flow at various Strouhal numbers on film cooling with cylindrical triple holes on a flat plate. The numerical results were compared with the numerical and experimental results for a single cylindrical hole on a flat plate. As a baseline, the effect of the main flow pulsation on contours of film cooling effectiveness and dimensionless temperature was first investigated for single-hole and triple-hole systems. Next, the time-averaged effectiveness of the film cooling was compared between these two systems. Instantaneous contours of $\eta$ on the wall were also investigated. Contours of the time-averaged velocity magnitude in the cooling hole were compared between the single-hole and triple-hole systems. Finally, Q-criterion iso-surfaces were compared and studied. The pulsations were sinusoidal, and the Strouhal number, non-dimensional frequency ranged from 0 to 1.6085. These numbers matched the pulsation frequency range of Seo et al. [9], allowing a comparison with their experimental results.

\section{Geometry, Boundary Conditions}

The geometry of the single-hole system, which was considered as the baseline, was obtained from the investigation of Seo et al. [9]. In their experimental apparatus, five cooling holes were positioned along a row. Here, the computational cost was reduced by applying periodic boundary conditions on the sidewalls [3]. The computational domains are displayed in Figure 1.

The triple holes consisted of two small holes located downstream of main hole (Figure 2b), as stated by Ely et al. [6]. In the triple-hole configuration, $D$ of the primary hole and the sister hole were calculated to match the total cross-sectional areas of the triple holes to the cross-sectional area of the single hole [3]. When the two small holes are located downstream of the main hole, the coolant jets generate an anti-counter-rotating vortex pair (anti-CRVP), and $\eta$ is increased through the generation of vortices interactions between the injectants and a reduction in the intensity of the main Counter Rotating Vortex Pair [3]. The hole-length-to-hole-diameter ratio $(L / D)$ for the single-hole system was 1.6, the ratios for the primary hole and each sister hole in the triple hole were 1.8 and 3.6, respectively, the injection angle of the coolant $(\alpha)$ was $35^{\circ}$. The turbulence intensity was set as $0.2 \%$ at main inlet (as reported for the experiment in [9]), and the velocity of the main flow was $10 \mathrm{~m} / \mathrm{s}$. The temperatures of the mainstream and injectant at the inlets were 300 and 293 Kelvin, respectively. The boundary conditions were specified in Table 1. Figure 2 presents schematics of the cooling holes in each case. 


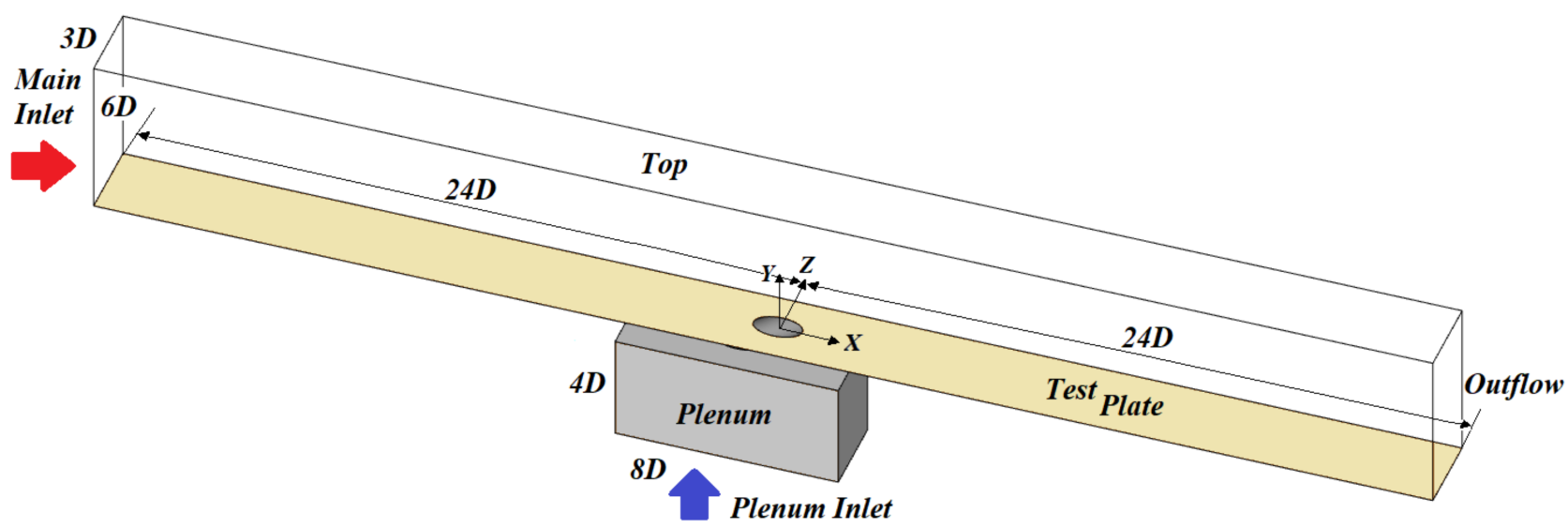

(a) Single-hole configuration

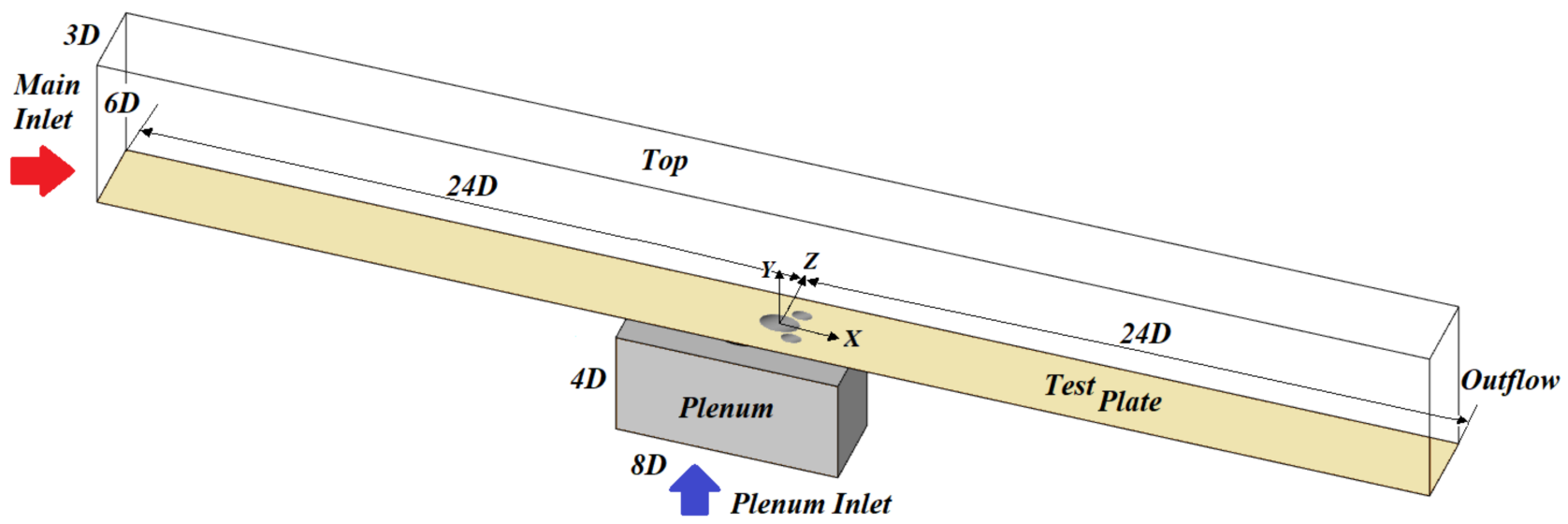

(b) Triple-hole configuration

Figure 1. Computational domains.

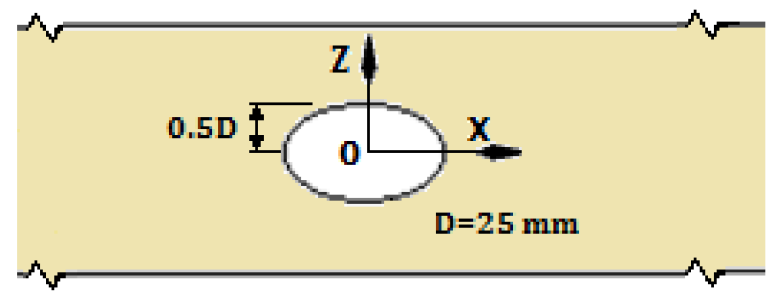

(a) Single-hole configuration

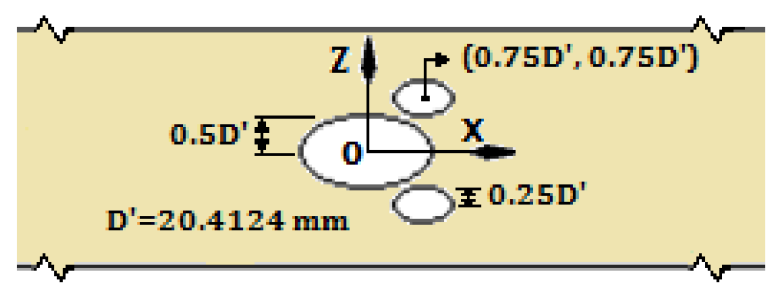

(b) Triple-hole configuration

Figure 2. Schematics of hole systems [3].

Table 1. Boundary conditions.

\begin{tabular}{cc}
\hline Surface & Boundary Condition \\
\hline Main inlet & Velocity inlet \\
Plenum inlet & Velocity inlet \\
Top & Symmetry \\
Test plate & Adiabatic wall \\
Outflow & Pressure outlet \\
Main sides & Periodic \\
Sides of plenum & Wall \\
Tube wall & Wall \\
\hline
\end{tabular}


The main flow velocity profile was uniform at the main inlet, similar to [9]. The main flow velocity at the inlet was:

$$
V_{\text {main inlet }}=A \sin (2 \pi \cdot f \cdot t)+10 \mathrm{~m} / \mathrm{s} \text {. }
$$

The values of the amplitude $A$ of the mainstream velocity pulsation considered here were identical to those used by Seo et al. [9]. The $A$ and frequency values at the different Strouhal numbers (Sr) are presented in Table 2. The Sr values for the single-hole system and the main and sister holes in the triple-hole configuration were identical, even though their $L / D$ values were different.

Table 2. Pulsation amplitude $A$ and frequency in Equation (1) at different Strouhal numbers (Sr).

\begin{tabular}{ccccc}
\hline Frequency $\mathbf{( H z )}$ & $\mathbf{0}$ & $\mathbf{2}$ & $\mathbf{1 6}$ & $\mathbf{3 2}$ \\
\hline$S r$ & 0 & 0.1005 & 0.8043 & 1.6085 \\
$A$ & 0 & 1.82 & 0.57 & 0.44 \\
\hline
\end{tabular}

The coolant velocity at the plenum inlet was calculated from the expression:

$$
V_{\text {plenum inlet }}=B \sin (2 \pi \cdot f \cdot t)+0.164 \mathrm{~m} / \mathrm{s} .
$$

While the values of the amplitude $B$ of the pulsations in the plenum inlet flow velocity were not specified by Seo et al. [9], they can be extracted from the $P_{2}-P_{1}$ variation plots for different frequencies, which are presented in their paper. Here, $P_{2}$ and $P_{1}$ are the static pressures at the inlet and outlet of the hole. The $B$ were acquired by matching $P_{2}-P_{1}$ plots to numerical calculations through trial and error. In Equation (2), 0.164 corresponds to the plenum inlet velocity on the steady state. Table 3 shows the $B$ values and frequencies at different Strouhal numbers. Increasing the pulsation frequency of the main flow increased the pressure difference between $P_{2}$ and $P_{1}$ since the phase shift increased [9], and this led to an increase in the amplitude of the coolant velocity pulsation, as is evident in Table 3.

Table 3. Pulsation amplitude $B$ and frequency in Equation (2) at different Strouhal numbers (Sr).

\begin{tabular}{ccccc}
\hline Frequency $\mathbf{( H z )}$ & $\mathbf{0}$ & $\mathbf{2}$ & $\mathbf{1 6}$ & $\mathbf{3 2}$ \\
\hline$S r$ & 0 & 0.1005 & 0.8043 & 1.6085 \\
$B$ & 0 & 0.04 & 0.05 & 0.16 \\
\hline
\end{tabular}

The Strouhal number is defined as $\mathrm{St}=\frac{2 \pi f L}{U_{C}}=\frac{L / U_{C}}{1 / 2 \pi f}$, and it denotes the ratio of the time needed for the coolant to pass through the hole to the duration of one pulsation cycle. If it is greater than 1 , the coolant is significantly affected by the pulsation during its passage through the hole [23]. Therefore, in this case, the trajectory of the injectant jet differs considerably from that in the steady state and the B value at $32 \mathrm{~Hz}$ sharply increases compared with that at 2 and $16 \mathrm{~Hz}$ (Table 3).

\section{Numerical Methods}

Numerical solutions were obtained by using ANSYS Fluent v.19 [3,24]. The CFD meshes were created in Pointwise v.18 [3,25]. CFD simulations were performed in the LES, Smagorinsky-Lilly model. The LES approach helps resolve large-scale eddies in turbulent flow and facilitates the modeling of small-scale eddies with length scales smaller than the grid spacing. The time step was $6.25 \times 10^{-6} \mathrm{~s}$, implying that the main flow convected the diameter of the single hole after 400 time steps [26,27]. In each time step, approximately 10 sub-iterations were executed to obtain well-resolved data [28]. The fluid was assumed as Newtonian and incompressible. The mainstream velocity was $10 \mathrm{~m} / \mathrm{s}$ and the mean 
injectant velocity was $5 \mathrm{~m} / \mathrm{s}$, showing that both velocities were far below Mach $=0.3[3,29]$. The governing equations were as shown in [24]:

$$
\begin{gathered}
\frac{\partial \rho}{\partial t}+\frac{\partial}{\partial x_{i}}\left(\rho \bar{u}_{i}\right)=0 \\
\frac{\partial(\rho \bar{u})}{\partial t}+\frac{\partial}{\partial x_{j}}\left(\rho \bar{u}_{i} \bar{u}_{j}\right)=\frac{\partial}{\partial x_{j}}\left[\mu\left(\frac{\partial \bar{u}_{i}}{\partial x_{j}}+\frac{\partial u_{j}}{\partial x_{i}}\right)-\frac{2}{3} \mu \frac{\partial \bar{u}_{l}}{\partial x_{l}} \delta_{i j}\right]-\frac{d \bar{p}}{d x_{i}}+\frac{\partial \tau_{i j}}{\partial x_{j}}
\end{gathered}
$$

Here, $\tau_{i j}$, which denotes the subgrid scale turbulent stress, required modeling by using the Boussinesq hypothesis, similar to RANS turbulence models, as follows:

$$
\tau_{i j}-\frac{1}{3} \tau_{k k} \delta_{i j}=-\mu_{t}\left(\frac{\partial \bar{u}_{i}}{\partial x_{j}}+\frac{\partial \bar{u}_{j}}{\partial x_{i}}\right)
$$

where $\mu_{t}$ represents the turbulent viscosity.

Figures 3 and 4 illustrate the meshes in the $x y$ and $y z$ planes, respectively [3]. The computational domains of the single-hole and triple-hole systems were composed of 2.04 and 2.69 million hexahedron cells, respectively [3]. Figure 5 illustrates a close-up of the mesh near hole. $P_{2}$ and $P_{1}$ represent the static pressures at the inlet and outlet of the hole, respectively.

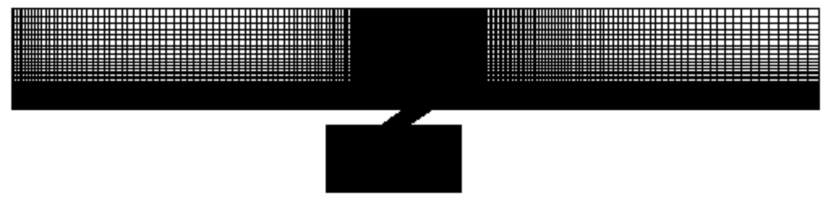

(a) Single-hole system

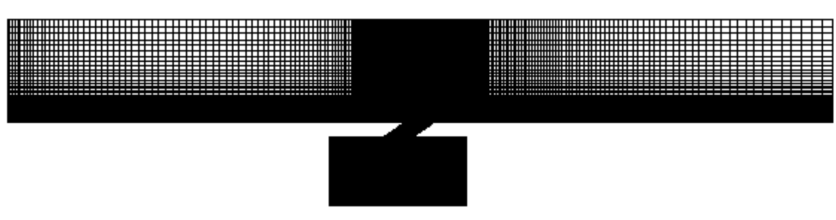

(b) Triple-hole system

Figure 3. Computational fluid dynamics grids in the $x y$ plane [3].

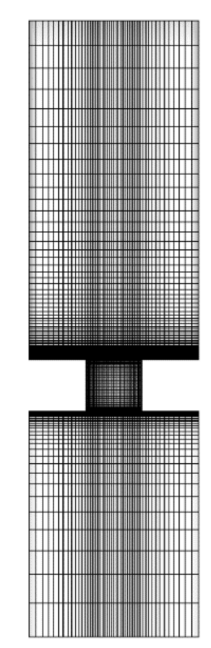

(a) Single-hole system

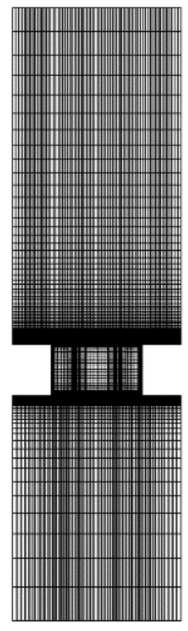

(b) Triple-hole system

Figure 4. Grids in the yz plane [21]. 


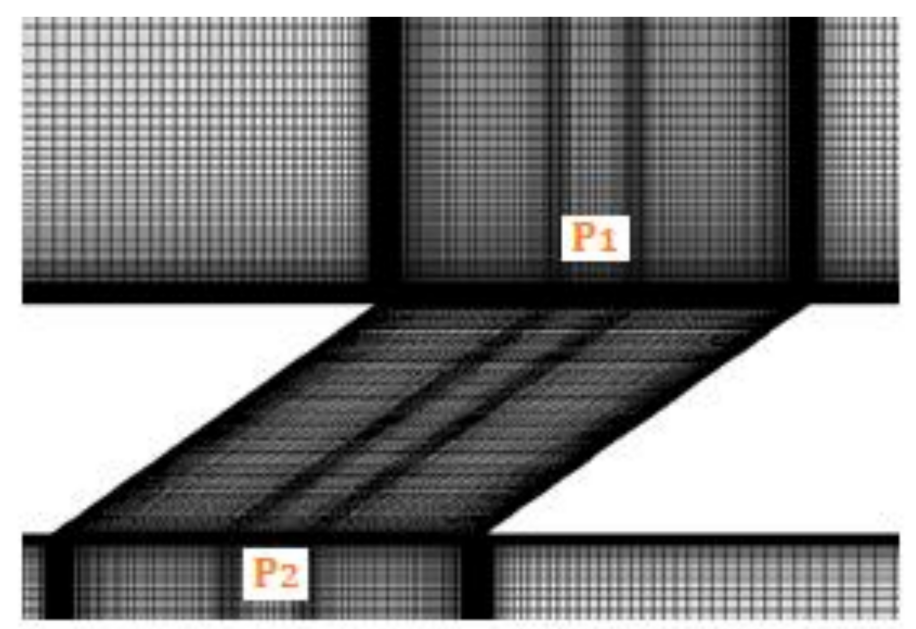

Figure 5. Close-up view of the grid near the injection region [3].

In [3], grid sensitivity tests were conducted in the steady case $(\mathrm{Sr}=0)$ and at the blowing ratio, $M$ of 0.5 for the single-hole and triple-hole systems.

\section{Results \& Discussion}

\subsection{Contours of Time-Averaged $\eta$ and Dimensionless Temperature}

Figures 6 and 7 show contours of time-averaged $\eta$ on the wall for the ordinary and triple hole, respectively, at various frequencies and Strouhal numbers. The contours for the single-hole system at 0 and $32 \mathrm{~Hz}$ are compared to the contours with the experimental data of Jung [30]. The effectiveness is slightly over-predicted by LES, however, the contours obtained in the experiment were similar to those predicted by the LES. The coolant's contact with the plate is hardly affected by an increase in the frequency from 0 to $16 \mathrm{~Hz}$. However, it is significantly reduced by the $32 \mathrm{~Hz}$ pulsation owing to increased mixing. The Strouhal number for the $32 \mathrm{~Hz}$ pulsation is 1.6085, and as stated earlier, if the Strouhal number is greater than 1 , the coolant is significantly affected by the pulsation during its passage through the hole. For each frequency of the pulsation, coolant spreading and contact with the test plate is considerably better in the triple-hole system compared with the ordinary hole system, as shown in Figures 6 and 7. The improvement is attributable to the anti-CRVPs generated by the sister holes, leading to weak main CRVP and weak entrainment of hot gas under the injectant. 


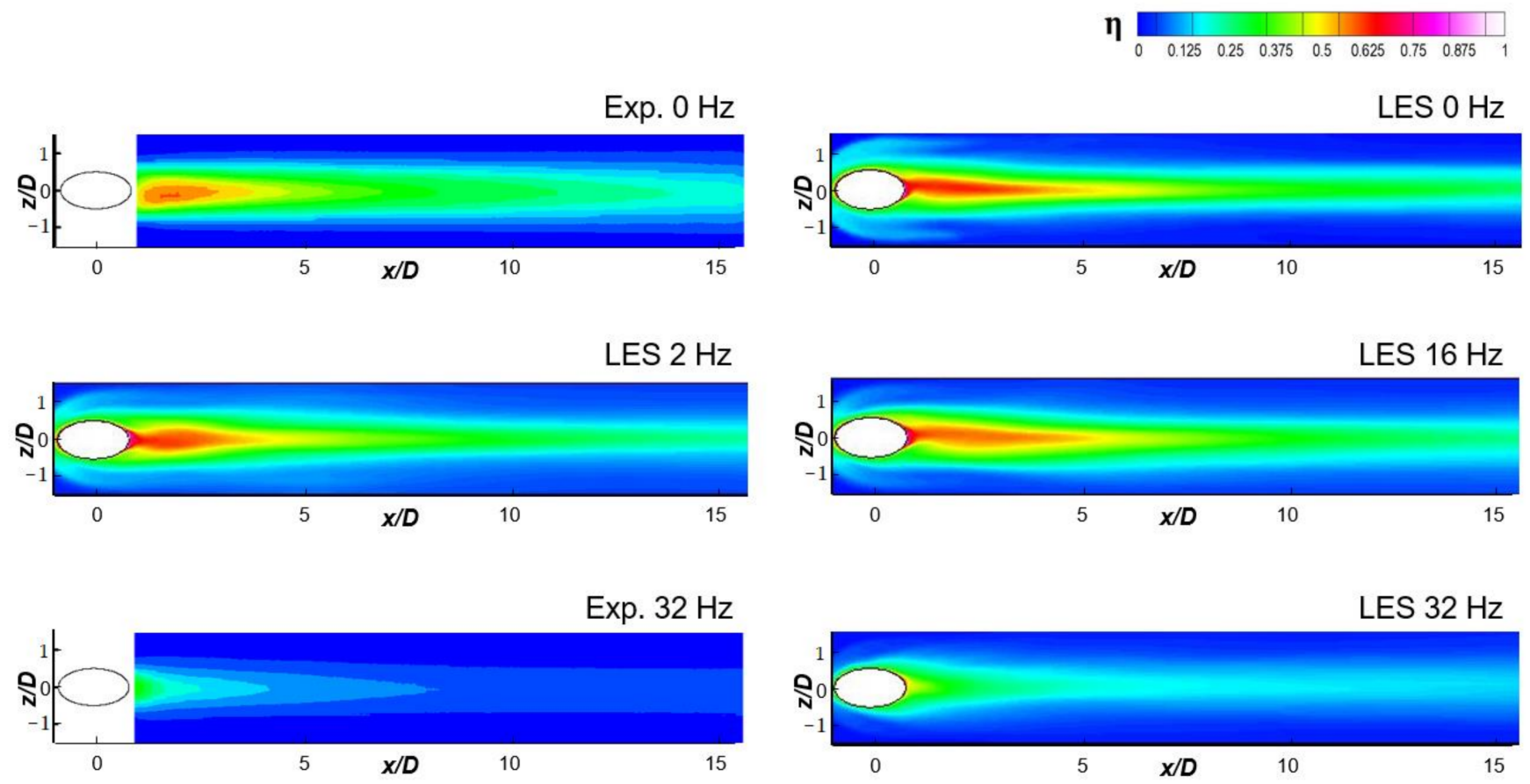

Figure 6. Contours of time-averaged $\eta$ on the wall for single-hole system for $0(\mathrm{Sr}=0), 2(\mathrm{Sr}=0.1005), 16(\mathrm{Sr}=0.8043)$ and $32 \mathrm{~Hz}(\mathrm{Sr}=1.6085)$.
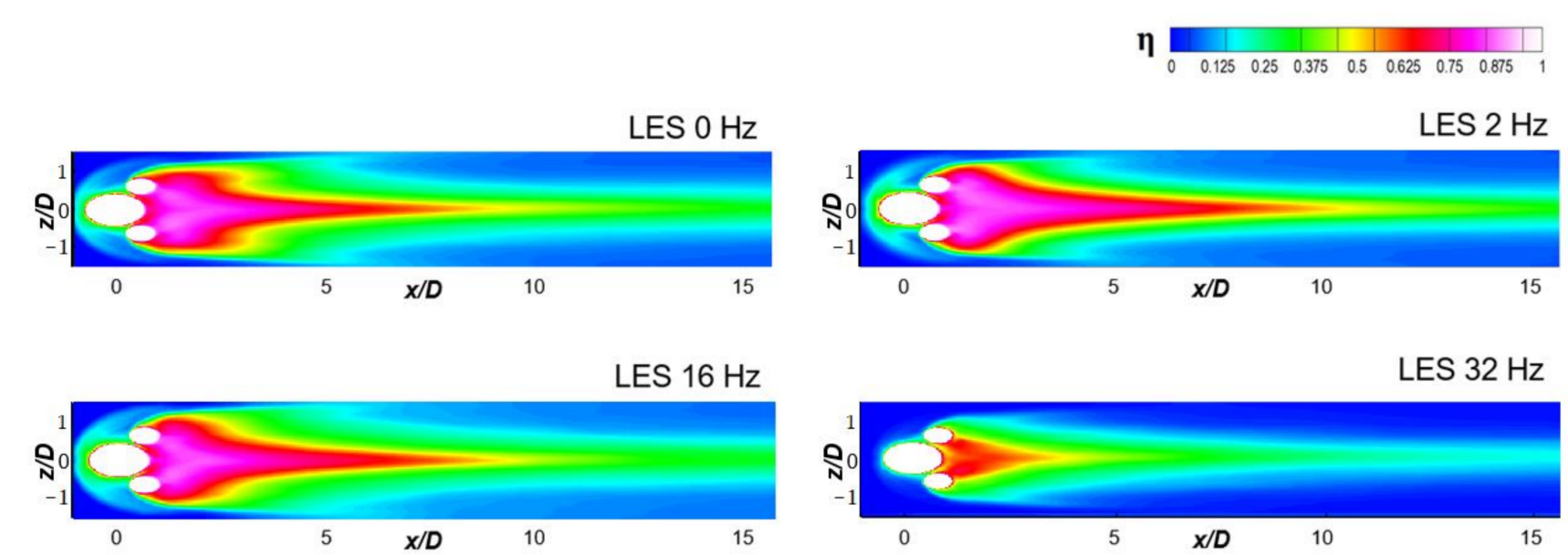

Figure 7. Contours of time-averaged $\eta$ on the wall for the triple hole for $0(\mathrm{Sr}=0), 2(\mathrm{Sr}=0.1005), 16(\mathrm{Sr}=0.8043)$ and $32 \mathrm{~Hz}$ $(\mathrm{Sr}=1.6085)$.

Figure 8 illustrates the cross-sectional dimensionless mean temperature contours at $x / D=2.5,5$, and 10 for 0 and $32 \mathrm{~Hz}$ pulsations and a comparison of the contours with the experimental data of Jung [30]. If there is $32 \mathrm{~Hz}$ pulsation in the flow, a smaller injectant comes in contact with the wall compared with at the case of $0 \mathrm{~Hz}$. However, as shown in the mean effectiveness contours of the film cooling on the wall in Figures 6 and 7, the coolant spreading and contact with the test plate is considerably better in the triple-hole compared with the single-hole system. 


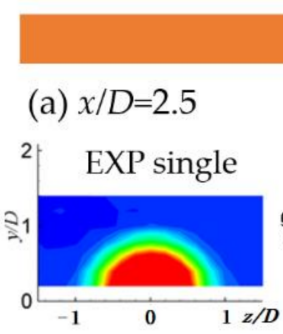

(b) $x / D=5$

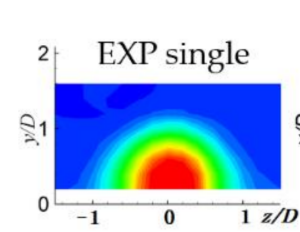

(c) $x / D=10$
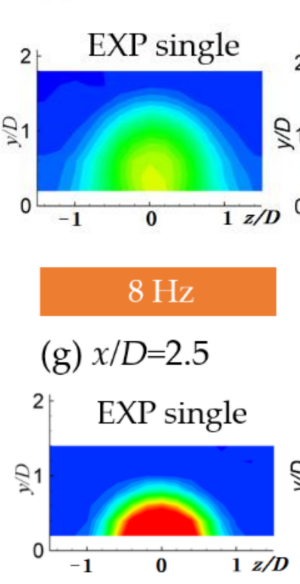

(h) $x / D=5$
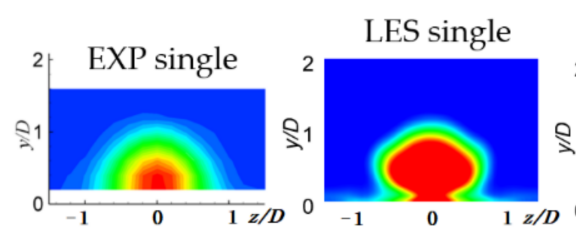

(i) $x / D=10$
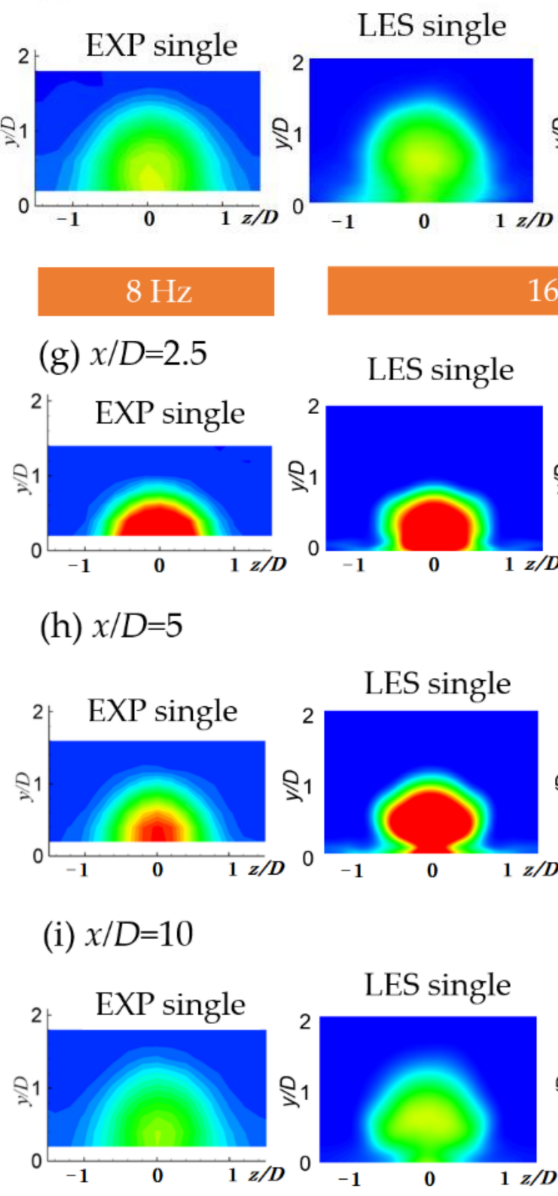

\section{$0 \mathrm{~Hz}$}

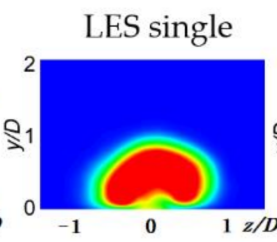

LES single
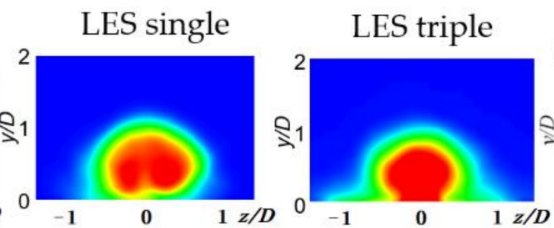

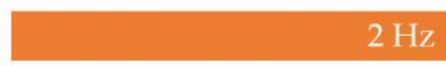

(d) $x / D=2.5$
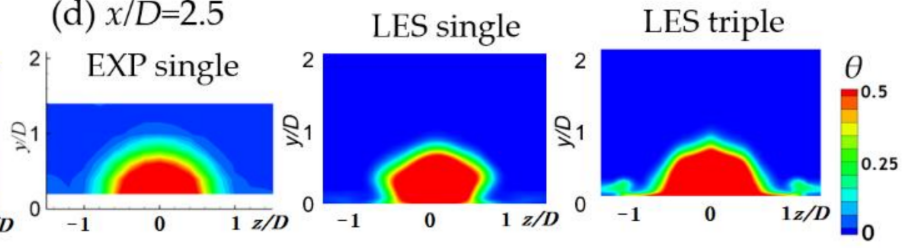

(e) $x / D=5$
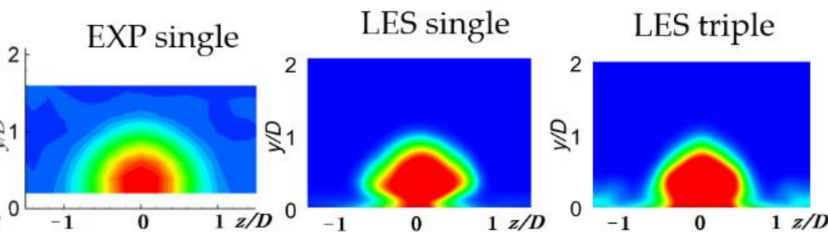

(f) $x / D=10$
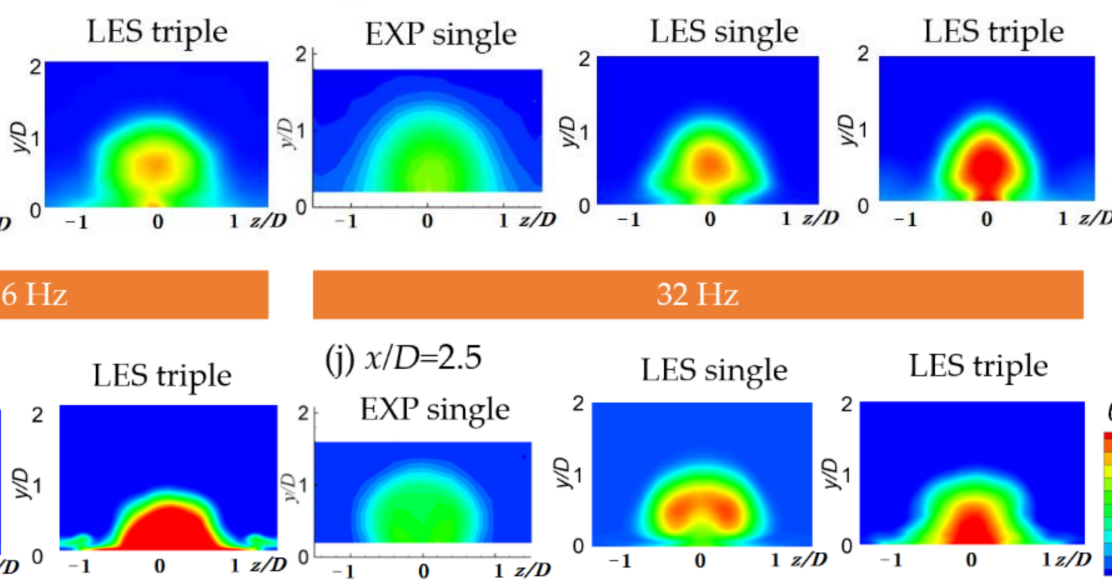

\section{$32 \mathrm{~Hz}$}
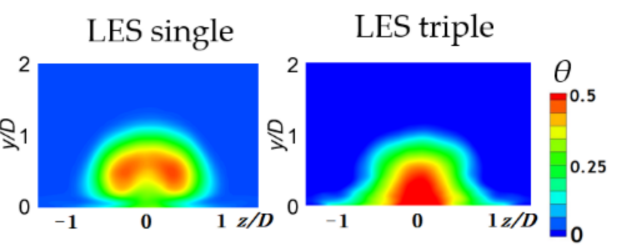

(k) $x / D=5$
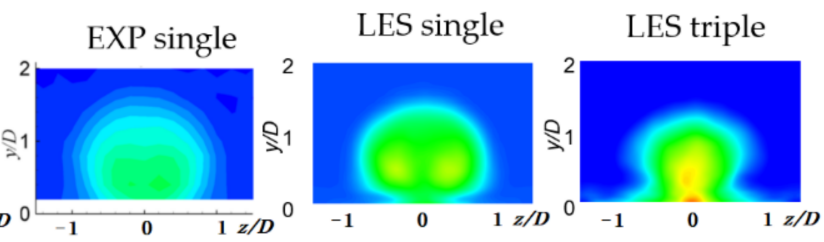

(l) $x / D=10$
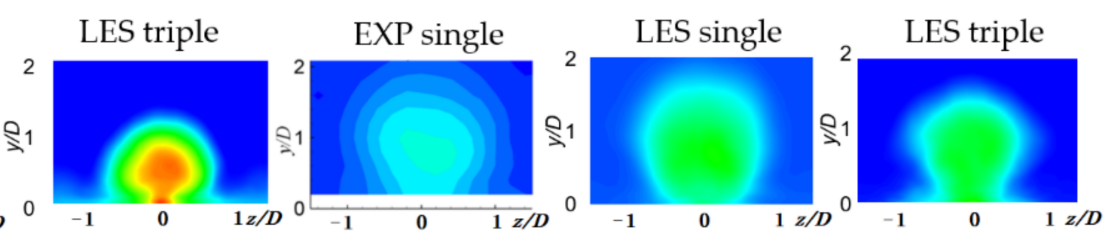

Figure 8. Cross-sectional time-averaged dimensionless temperature distributions at $M=0.5$.

As shown in the contours of the experimental data in Figure 8, there is no data for the dimensionless temperature near the test plate because it could not be measured through cold-wire measurement. However, except near the test plate, the contours obtained in the experiment were similar to those predicted by the LES. The small difference between the LES results and the experimental data is because the experimental data were measured using an apparatus with a single hole with $L / D=4$ while the LES results were obtained with a single hole with $L / D=1.6$. The dimensionless temperature near the plate decreased as the mainstream flow travelled downstream, and $x / D$ increased because of the intense 
mixing of the mainstream flow and coolant jet by the turbulence. Thus, at $x / D=10$, the temperatures near the wall in the triple hole configuration and the single cooling hole configuration becomes similar because of the strong mixing. Figures 9 and 10 show the contours for the time-averaged dimensionless temperature at $x / D=2$ for the single-hole and triple-hole systems for $0,2,16$, and $32 \mathrm{~Hz}$ pulsations. The anti-Counter Rotating Vortex Pairs weakened the main Counter Rotating Vortex Pair and reduced the lift off of the coolant jet, decreasing the entrainment of the hot gas of main flow under the injectant [3]. Therefore, the temperature near the test plate remained similar to the temperature of the coolant-core part and the film cooling effectiveness increased. Figures 9 and 10 display cross-sectional contours of the mean dimensionless temperature and the mean velocity vectors at $x / D=2$ for the single-hole and triple-hole systems, respectively. As shown in Figure 10d, the entrainment of the hot gas of the main flow under the injectant in the triple cooling hole at $32 \mathrm{~Hz}$ was weaker than the entrainment in the single hole at $32 \mathrm{~Hz}$ in Figure 9d. The mean velocity vectors were superimposed on the cross-sectional views of mean contours of the temperature at $x / D=2$. In the triple cooling hole, the velocity vectors going upward were less concentrated at $z=0$ compared with the single cooling hole, and the liftoff of the injectant was weakened by the generation of anti-CRVPs.

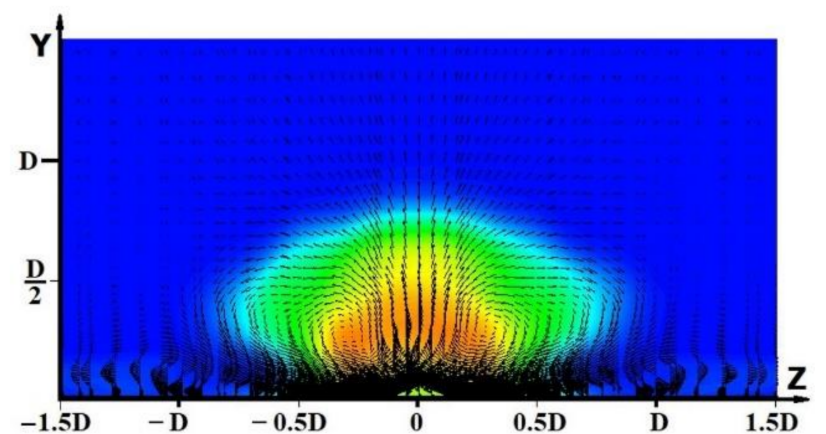

(a) $0 \mathrm{~Hz}, \mathrm{Sr}=0$

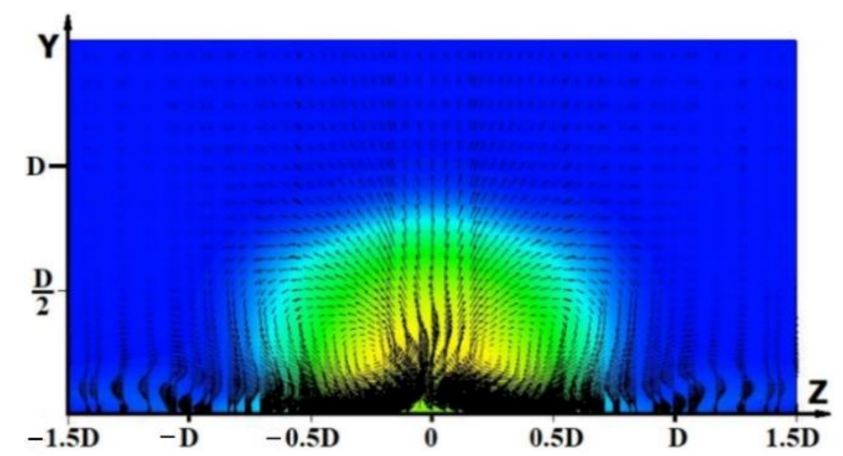

(c) $16 \mathrm{~Hz}, \mathrm{Sr}=0.8043$

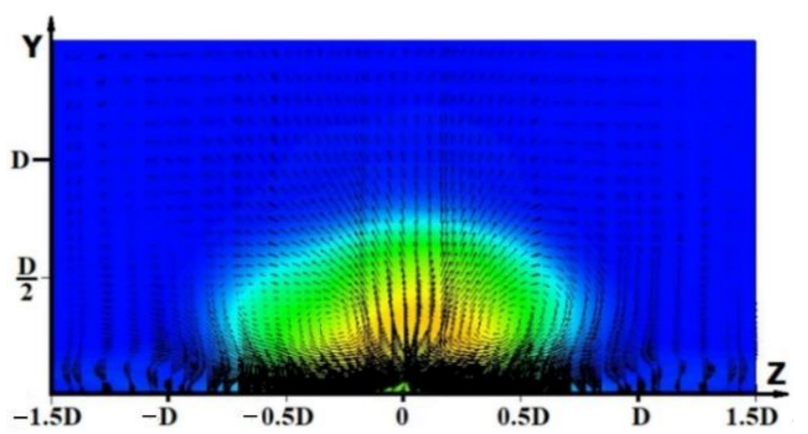

(b) $2 \mathrm{~Hz}, \mathrm{Sr}=0.1005$

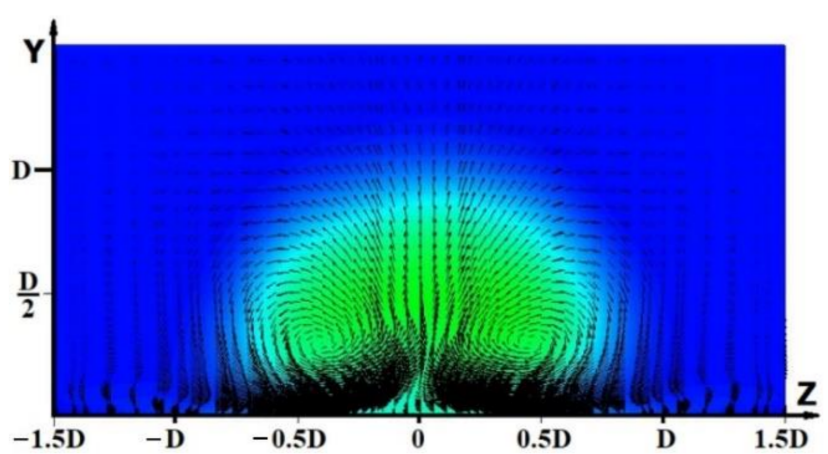

(d) $32 \mathrm{~Hz}, \mathrm{Sr}=1.6085$

$\Theta$

$\begin{array}{lllllllll}0.125 & 0.25 & 0.375 & 0.5 & 0.625 & 0.75 & 0.875 & 1\end{array}$

Figure 9. Contours of time-averaged dimensionless temperature and velocity vectors at $x / D=2$ for the single cooling hole system: (a) $0 \mathrm{~Hz}, \mathrm{Sr}=0$; (b) $2 \mathrm{~Hz}, \mathrm{Sr}=0.1005$; (c) $16 \mathrm{~Hz}, \mathrm{Sr}=0.8043$; and (d) $32 \mathrm{~Hz}, \mathrm{Sr}=1.6085$. 


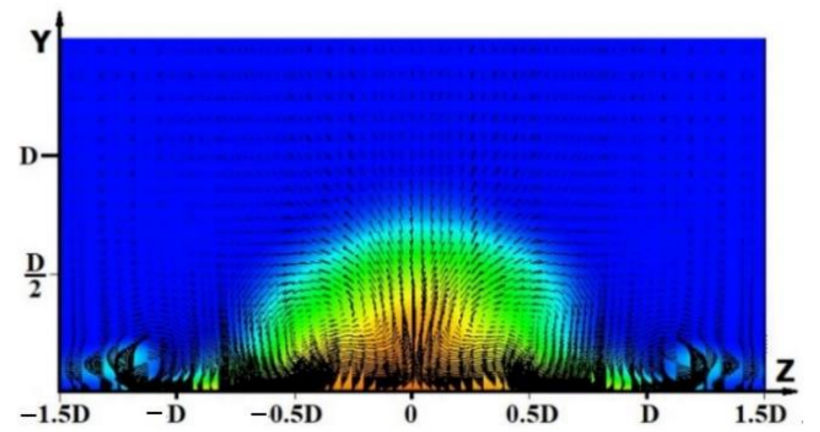

(a) $0 \mathrm{~Hz}, \mathrm{Sr}=0$

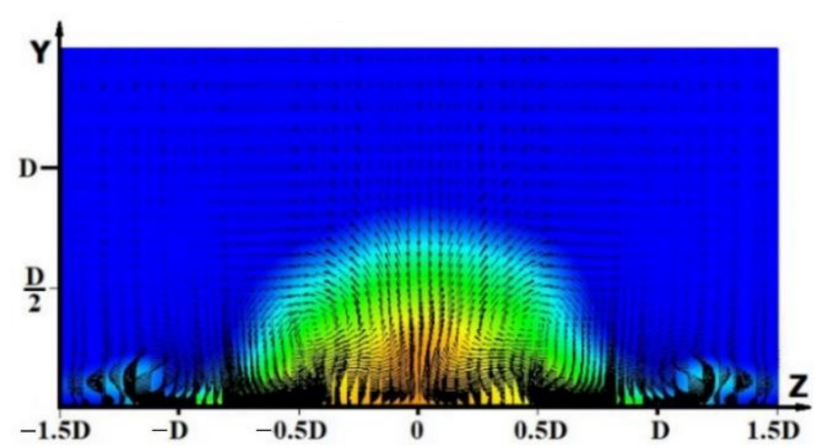

(c) $16 \mathrm{~Hz}, \mathrm{Sr}=0.8043$

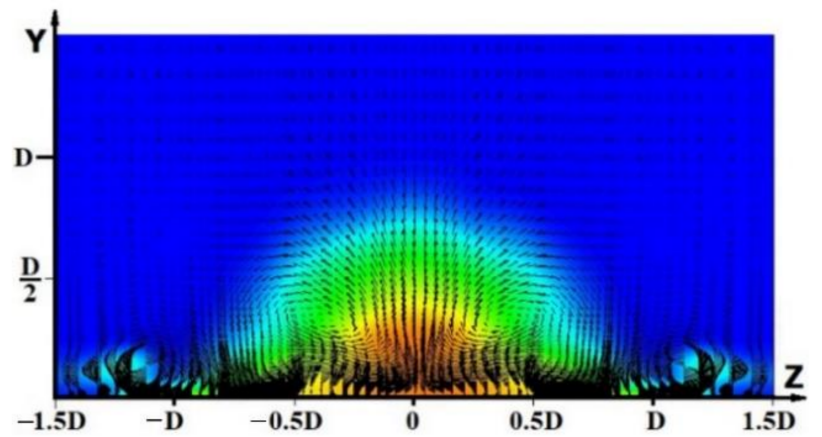

(b) $2 \mathrm{~Hz}, \mathrm{Sr}=0.1005$

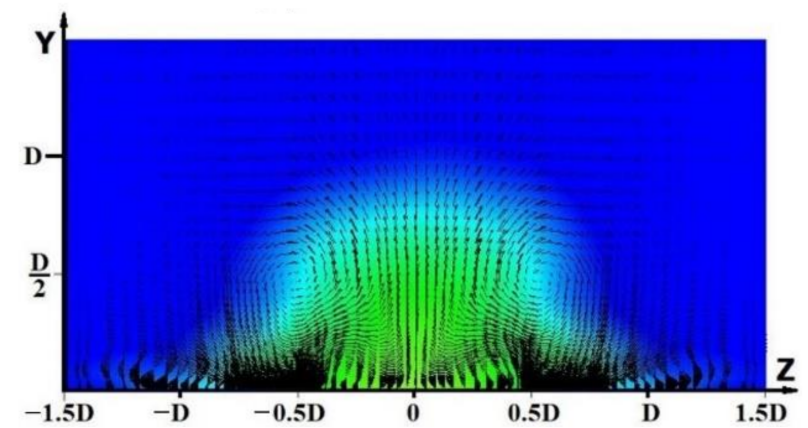

(d) $32 \mathrm{~Hz}, \mathrm{Sr}=1.6085$

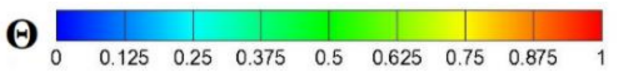

Figure 10. Contours of time-averaged dimensionless temperature and velocity vectors at $x / D=2$ for the triple-hole system (a) $0 \mathrm{~Hz}, \mathrm{Sr}=0$; (b) $2 \mathrm{~Hz}, \mathrm{Sr}=0.1005$; (c) $16 \mathrm{~Hz}, \mathrm{Sr}=0.8043$; and (d) $32 \mathrm{~Hz}, \mathrm{Sr}=1.6085$.

\subsection{Time-Averaged Effectiveness}

When the mainstream flow and injectant oscillated in the single and triple-hole configuration, the spanwise-averaged effectiveness showed identical trends. As shown in Figure 11, increasing the flow frequency reduced $\eta_{m}$. For the single hole, when the frequency was increased from 0 to 2,16, and $32 \mathrm{~Hz}$ and the Strouhal number (Sr) from 0 to $0.1005,0.8043$, and 1.6085 , the spanwise-averaged film cooling effectiveness decreased by approximately $11 \%, 12 \%$, and $45 \%$, respectively. For the triple-hole system, identical increases in the frequency and Strouhal number decreased the spanwise-averaged effectiveness by approximately $4 \%, 5 \%$, and $39 \%$, respectively. When the frequency was increased from 16 to $32 \mathrm{~Hz}$, the sharp decrease in $\eta_{m}$ was because when the Strouhal number is greater than 1 , the trajectory of the injectant is considerably different from that in the steady state and the B value in Equation (2) at $32 \mathrm{~Hz}$ sharply increases compared with that at 2 and $16 \mathrm{~Hz}$. Nevertheless, the triple-hole system showed higher effectiveness than the single-hole system, regardless of the frequency and Strouhal number, as shown in Figure 12. Experimental data [9] were available only for the single-hole system, and $\eta_{m}$ obtained by the LES matched well with the experimental data, even though the LES results were slightly underestimated. Figure 13 depicts the ratios of $\eta_{m}$ in the triple-hole configuration to $\eta_{m}$ in the single-hole, for various Strouhal numbers; the ratios were obtained through LES analyses. 


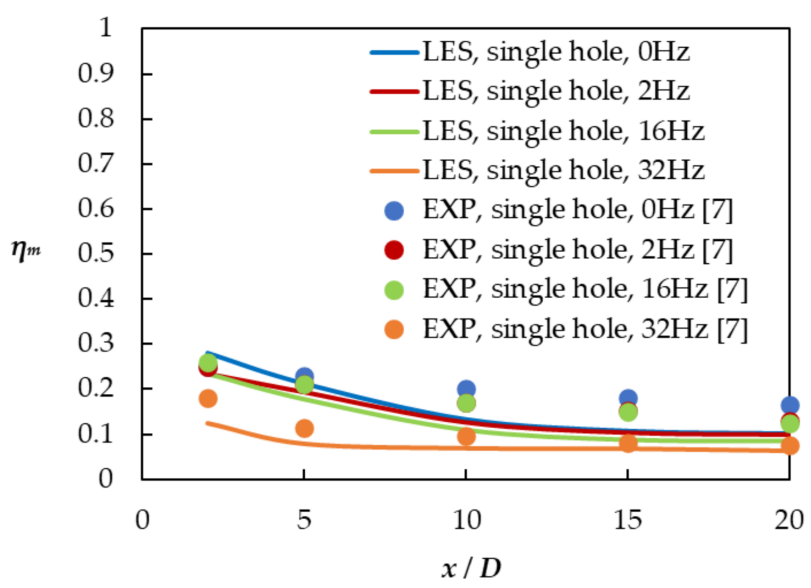

(a) Single-hole system

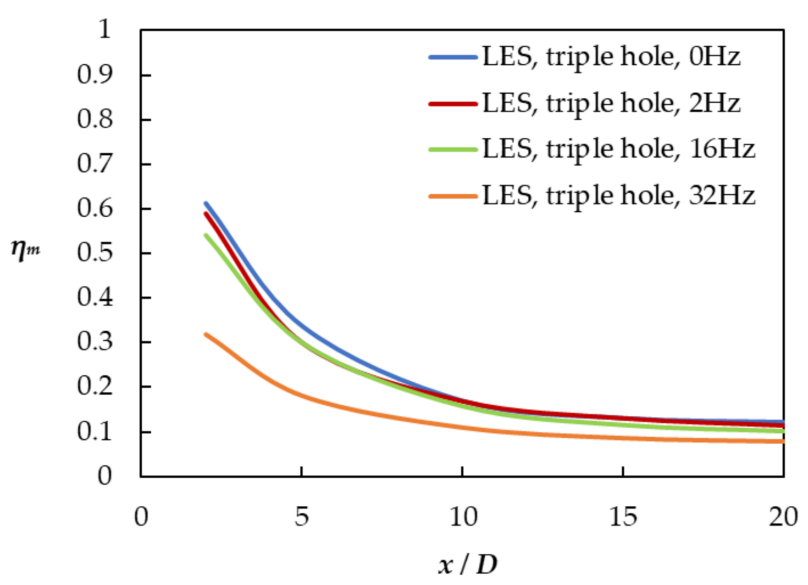

(b) Triple-hole configuration

Figure 11. $\eta_{m}$ under unsteady conditions.

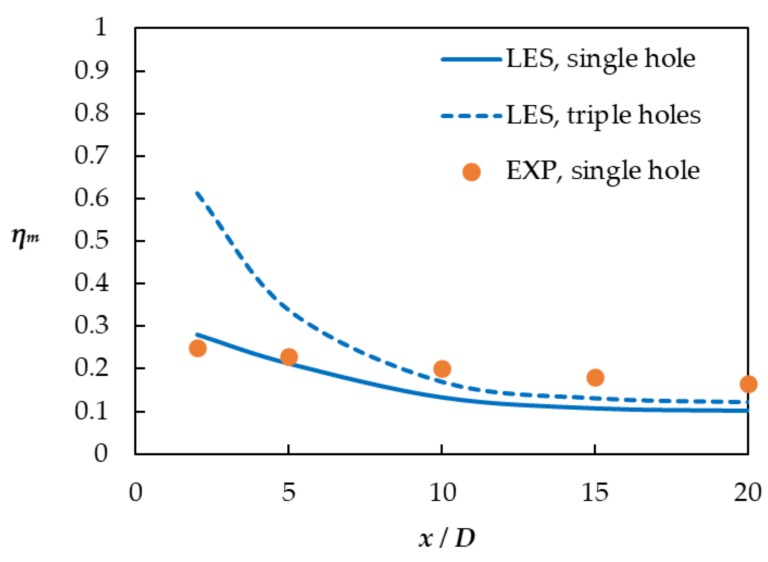

(a) $0 \mathrm{~Hz}, \mathrm{Sr}=0$

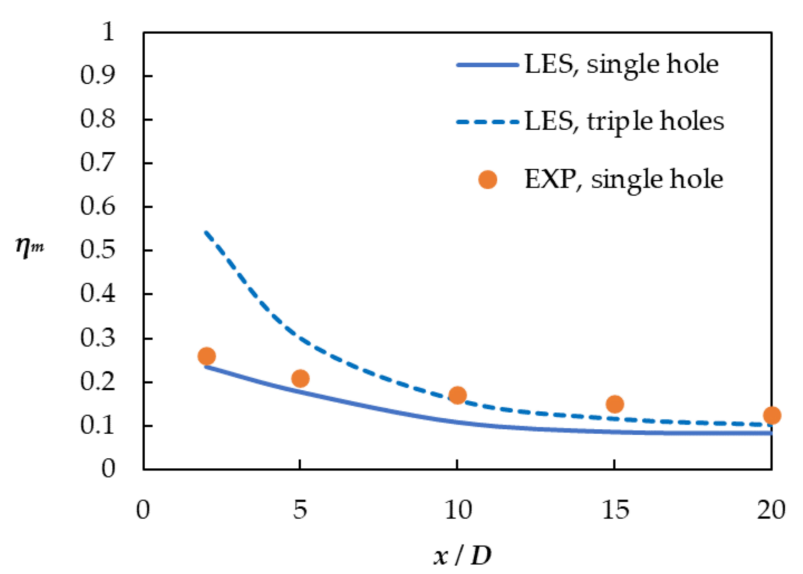

(c) $16 \mathrm{~Hz}, \mathrm{Sr}=0.8043$

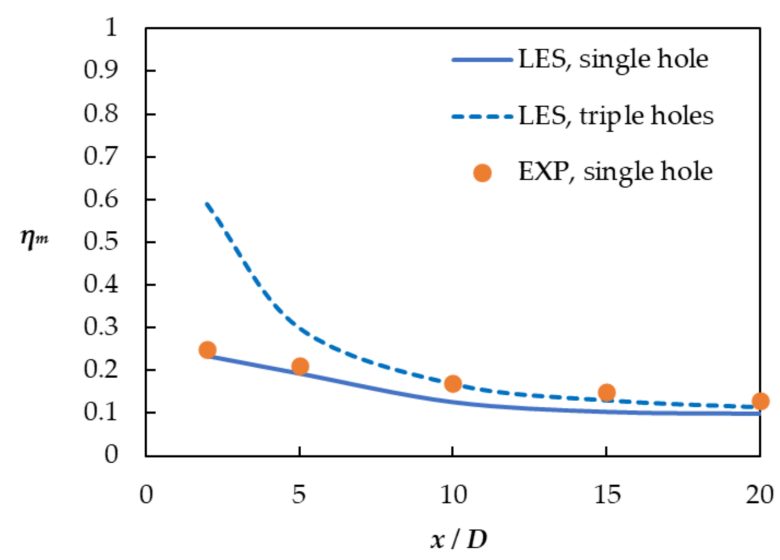

(b) $2 \mathrm{~Hz}, \mathrm{Sr}=0.1005$

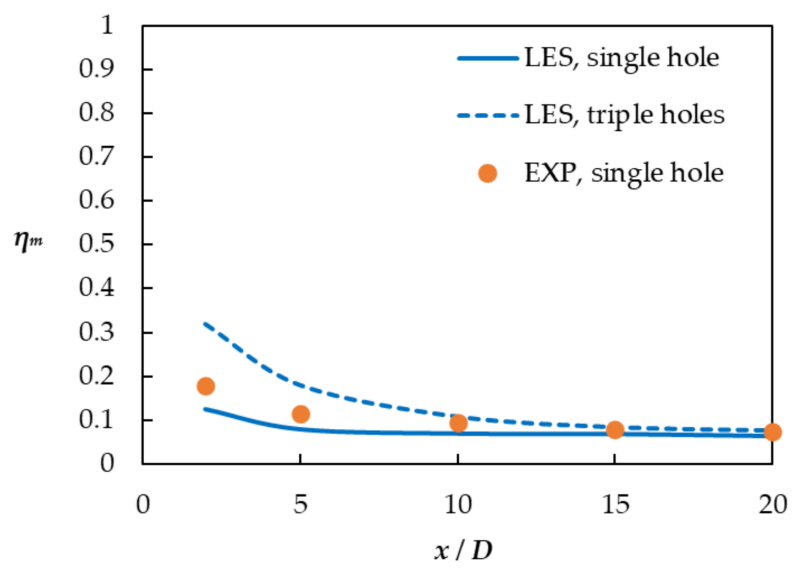

(d) $32 \mathrm{~Hz}, \mathrm{Sr}=1.6085$

Figure 12. Laterally-averaged effectiveness under unsteady conditions: (a) $0 \mathrm{~Hz}, \mathrm{Sr}=0$; (b) $2 \mathrm{~Hz}, \mathrm{Sr}=0.1005$; (c) $16 \mathrm{~Hz}$, $\mathrm{Sr}=0.8043$; and $(\mathbf{d}) 32 \mathrm{~Hz}, \mathrm{Sr}=1.6085$. 


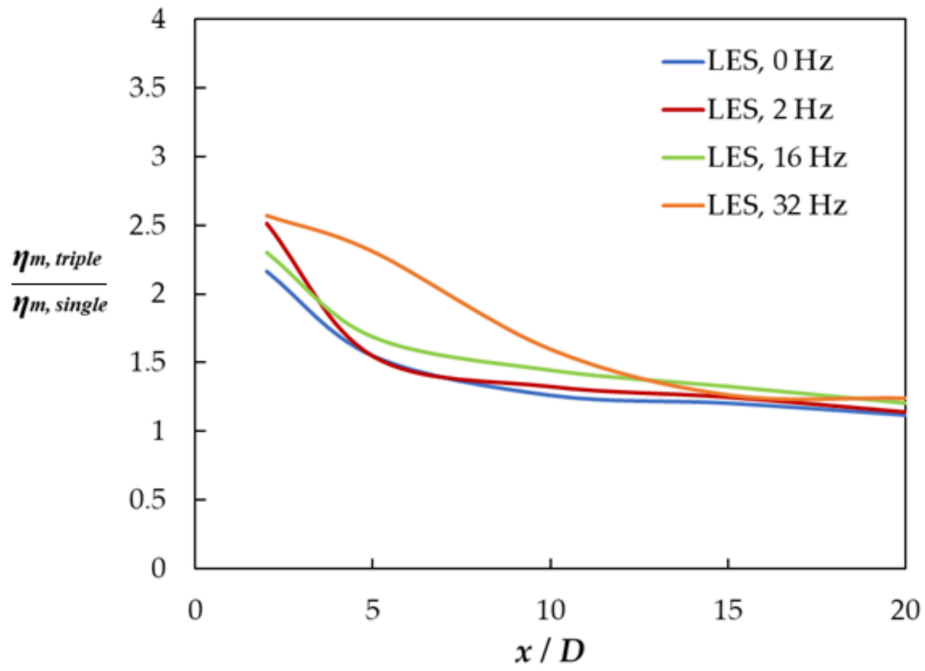

Figure 13. Ratios of $\eta_{m \text {,triple }}$ to $\eta_{m \text {,single }}$ at $0 \mathrm{~Hz}(\mathrm{Sr}=0), 2 \mathrm{~Hz}(\mathrm{Sr}=0.1005), 16 \mathrm{~Hz}(\mathrm{Sr}=0.8043)$, and $32 \mathrm{~Hz}(\mathrm{Sr}=1.6085)$.

Increasing the pulsation frequency and Strouhal number increased the ratio of the spanwise-averaged effectiveness. While the increase was small between $0 \mathrm{~Hz}(\mathrm{Sr}=0)$ and $16 \mathrm{~Hz}(\mathrm{Sr}=0.8043)$, it was sharp from $16 \mathrm{~Hz}(\mathrm{Sr}=0.8043)$ to $32 \mathrm{~Hz}(\mathrm{Sr}=1.6085)$. This indicated that under unsteady main flows and higher pulsation frequencies and Strouhal numbers, configuring a primary hole and two small holes is better than configuring a single hole for film cooling. The coolant injection generated large disturbances in the main flow at $32 \mathrm{~Hz}$ because under these conditions, the amplitude of the cooling air velocity pulsation increased remarkably at the inlet of the plenum, as evident in Table 3, and a high jet liftoff was generated as mentioned earlier. Anti-CRVPs reduce the intensity of the main CRVP more effectively when high disturbances are generated in the main flow. Additionally, the ratio of the spanwise-averaged effectiveness was almost independent of the Strouhal number at $x / D>13$ because the disturbances induced by the coolant jet pulsation weakened in this $x / D$ range, reducing the film cooling effectiveness of the anti-CRVPs.

\subsection{Instantaneous Effectiveness Contours on the Wall}

Figure 14 shows contours of instantaneous film cooling effectiveness on the test plate obtained through LES at 0 and $32 \mathrm{~Hz}$. The variable $t^{*}$ in Figure $14 \mathrm{a}, \mathrm{b}$ represents the dimensionless time defined by the mainstream flow velocity and hole diameter, and it indicates $t^{*}=1$ as the time required for the main flow to travel across the hole diameter. Figure 14a shows LES contours of instantaneous $\eta$ for the single-hole configuration under steady conditions at $t^{*}=2,4,6,8$, and 10, and each contour does not show a big difference. Figure 14c,d shows the LES-derived instantaneous effectiveness of the film cooling on the test plate for each phase with $32 \mathrm{~Hz}$ flow pulsation. The figures show the periodic formation of a coolant covering on the plate when the coolant injection velocity varies periodically. When the frequency of the mainstream and coolant jet was increased, higher jet liftoff was generated, leading to the coolant periodically touching the test plate; the coolant could not cover the test plate efficiently as it did for $0 \mathrm{~Hz}$. However, the contours for the triple-hole system showed a wider coolant spread in the spanwise direction than those for the single hole. Furthermore, the coolant covered the test plate much better through the generation of anti-CRVPs. The horseshoe vortex is shown in all contours for $0 \mathrm{~Hz}$ in Figure 14a,b, whereas the vortex is visible in the contours periodically at $32 \mathrm{~Hz}$ pulsation in Figure 14c,d. The horseshoe vortex is formed when the vortices generated in the boundary layer of the main flow are blocked by coolant injection and roll-up [31,32]. Therefore, for $32 \mathrm{~Hz}$ pulsation, when the injectant velocity is high magnitude velocity part of the cycle, the vortices in the boundary layer of the main flow are blocked by the injection of the coolant, resulting in a horseshoe vortex being generated as can be seen at $t / \tau=0.2$ in 
Figure $14 \mathrm{c}, \mathrm{d}$. If the injectant velocity is low, the horseshoe vortex is not generated easily, and it is not clearly visible on the contour.

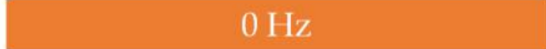

(a) LES, Single hole, $0 \mathrm{~Hz}$

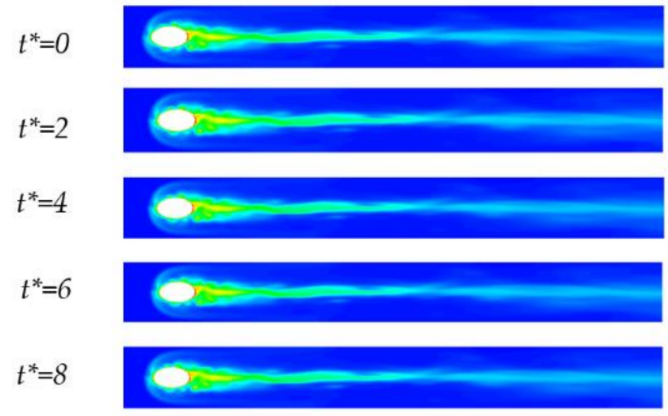

(b) LES, Triple hole, $\mathrm{OHz}$

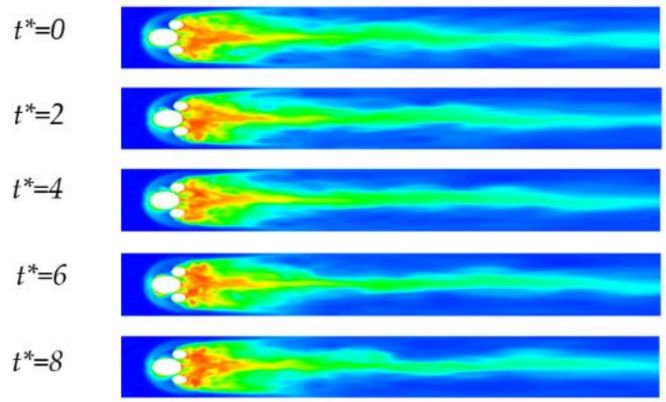

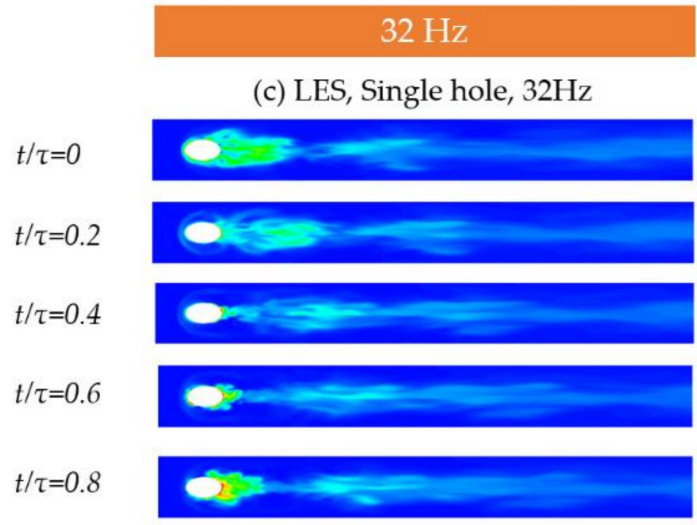

(d) LES, Triple hole, $32 \mathrm{~Hz}$

$t / \tau=0$

$t / \tau=0.2$

$t / \tau=0.4$

$t / \tau=0.6$

$t / \tau=0.8$

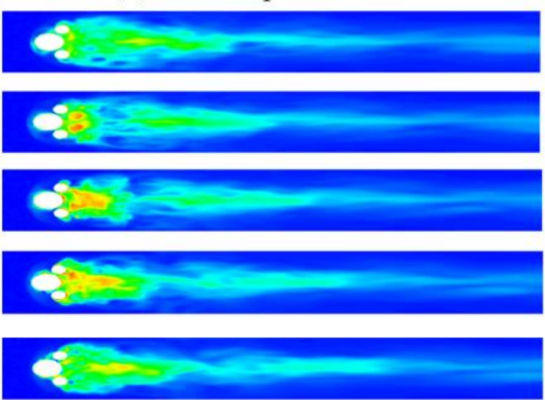

Figure 14. Contours of instantaneous $\eta$ on the wall obtained by an LES: (a) Single hole at $0 \mathrm{~Hz}$, (b) triple holes at $0 \mathrm{~Hz}$, (c) single hole at $32 \mathrm{~Hz}$, and (d) triple holes at $32 \mathrm{~Hz}$.

\subsection{Time-averaged Velocity Magnitude in the Hole}

Figure 15 illustrates time-averaged velocity magnitude contours at the hole exit for 0 and $32 \mathrm{~Hz}$, obtained by an LES, and Figure 16 depicts contours of the time-averaged velocity magnitude in the hole in the $\mathrm{z}=0$ plane. The ratio of $L$ to $D$ in the single-hole configuration was set as 1.6, and the ratios of $L$ to $D$ for the primary and sister hole in the triple hole were 1.8 and 3.6, respectively. Therefore, in the triple hole, the mean velocity magnitude at the main hole exit shows distinct high and low momentum regions because of the small $L / D$ value, while that at the sister hole exit indicates a more developed and uniform flow velocity [3]. The cooling jet with more uniform velocity is better for $\eta$ due to less injectant lift off. Therefore, small hole in the triple-hole system improve $\eta$ through the generation of an anti-CRVP and by increasing the $L / D$ ratios. The same concept holds for $32 \mathrm{~Hz}$ pulsation. When the flow oscillates at $32 \mathrm{~Hz}$, the contours of time-averaged velocity magnitude at the hole exit of the single-hole system show more uniform injectant velocity magnitude than those at $0 \mathrm{~Hz}$ since the amplitude of the coolant velocity at the hole exit varies periodically and thereby generates large disturbances and mixing. However, the contours of the mean velocity magnitude in the sister holes show flow that is more developed than that in the main hole, even at $32 \mathrm{~Hz}$ pulsation. Thus, the small holes increase $\eta$ through the formation of anti-Counter Rotating Vortex Pair and by increasing the $\mathrm{L} / D$ ratios. In Figure $16 \mathrm{~d}$,e, the main flow can be inferred to invade into the windward side of the hole exit because when the flow oscillates at $32 \mathrm{~Hz}$, the hot main flow in the hole reverses periodically, which is detrimental to the hole material. 


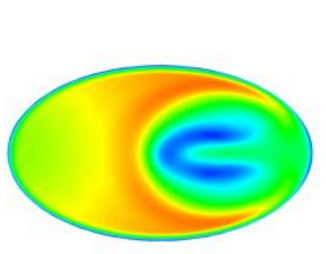

(a) Single hole at $0 \mathrm{~Hz}$

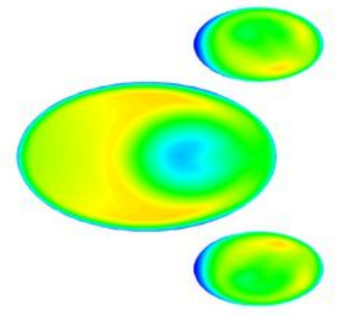

(b) Triple holes at $0 \mathrm{~Hz}$

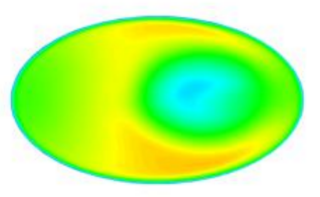

(c) Single hole at $32 \mathrm{~Hz}$

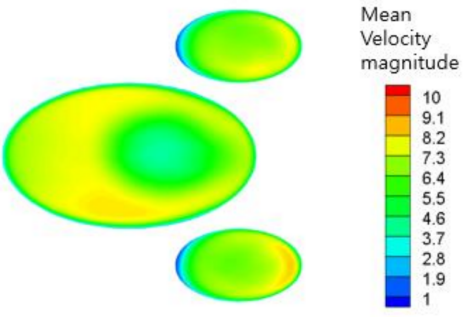

(d)Triple holes at $32 \mathrm{~Hz}$

Figure 15. Mean velocity magnitude at the hole exit.
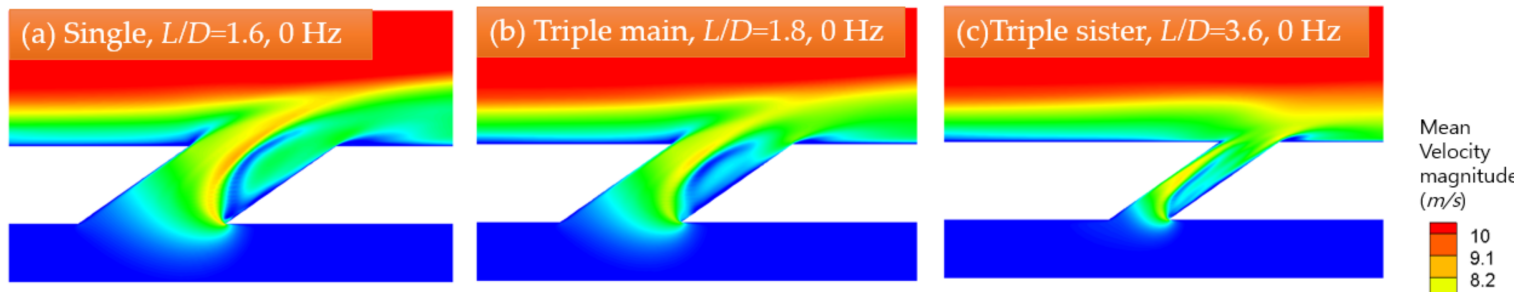
magnitud $(\mathrm{m} / \mathrm{s})$
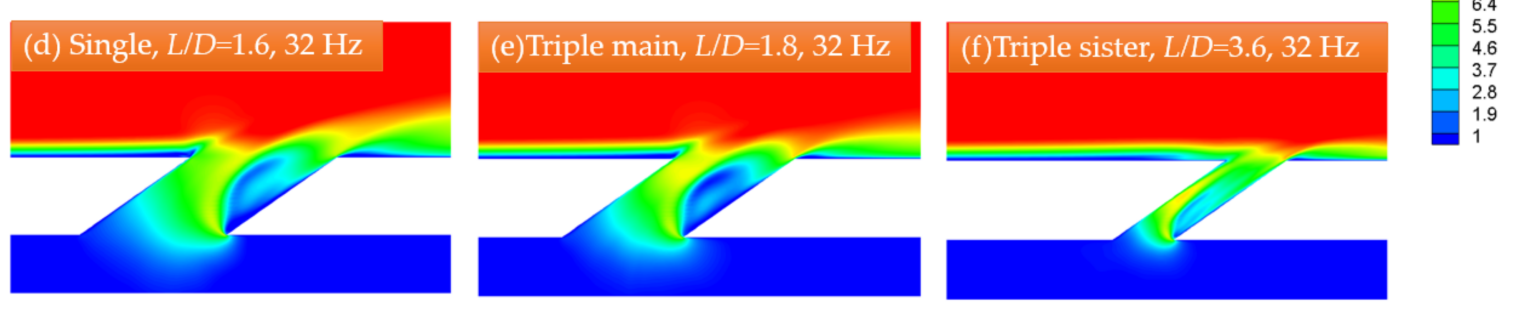

Figure 16. Contours of time-averaged velocity magnitude in the hole at the centerline.

Figure 17a,b shows profiles of the mean coolant velocity magnitude at the hole exit in the streamwise direction for $L / D$ ratios around 1.6 and 4 , respectively. As stated earlier, the mean velocity magnitude at the hole exit shows large variations for $L / D$ values of 1.6 or 1.8 , while that for $L / D$ values of 3.6 or 4 is more uniform. Specifically, for the $L / D$ value of 1.8 , the mean velocity magnitude shows smaller variations compared with those for the $L / D$ value of 1.6. Further, when there is $32 \mathrm{~Hz}$ pulsation in the flow, the mean velocity magnitude becomes more uniform than that in the steady state. The difference in the coolant velocity magnitude between the LES results and the experimental data for the leeward side of the hole $(0<x / D<0.8)$ is attributed to the experimental operating conditions [9]. The experimental data were measured in the absence of the main flow.

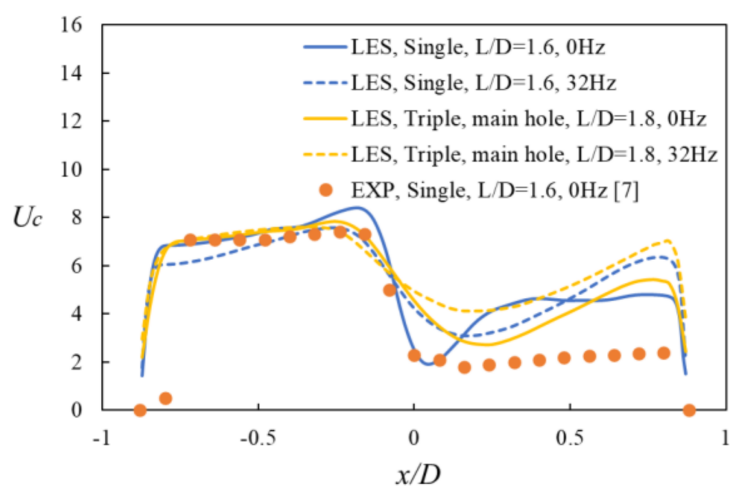

(a) $\mathrm{L} / \mathrm{D}$ is around 1.6

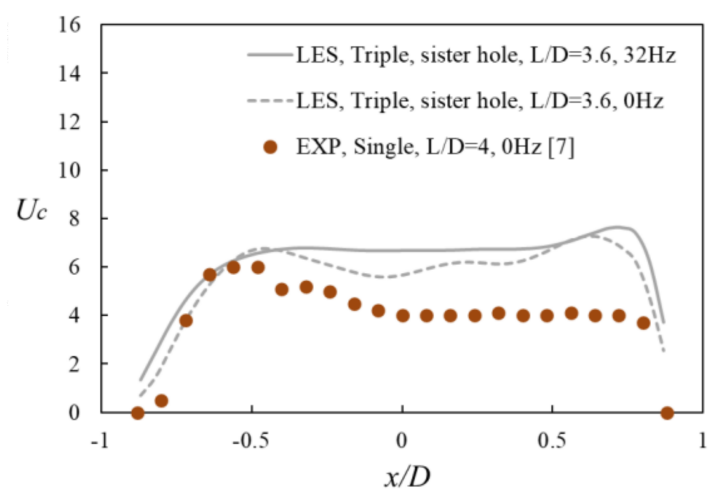

(b) $\mathrm{L} / \mathrm{D}$ is around 4

Figure 17. Profiles of the mean coolant velocity magnitude at the hole exit in the streamwise direction. 


\subsection{Q-Criterion Contour}

Q-criterion represents the dominance of the rotation rate over the strain rate [33,34]. The instantaneous contours of the Q-criterion for the single and triple hole configurations at 0 and $32 \mathrm{~Hz}$ are shown in Figure 18.

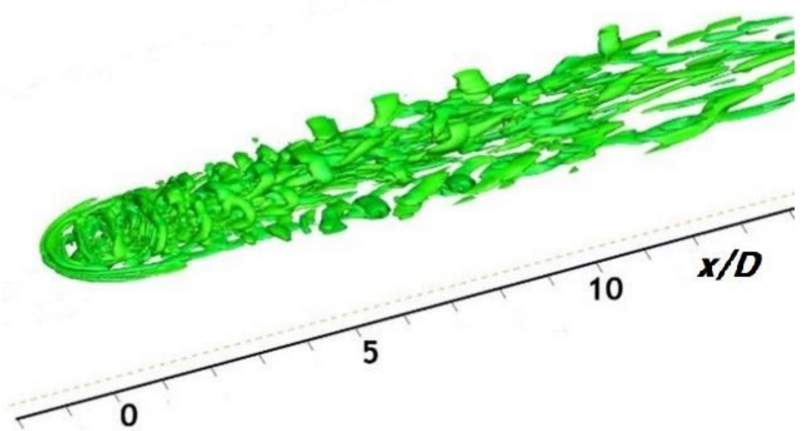

(a) Single-hole system at $0 \mathrm{~Hz}$ [3]

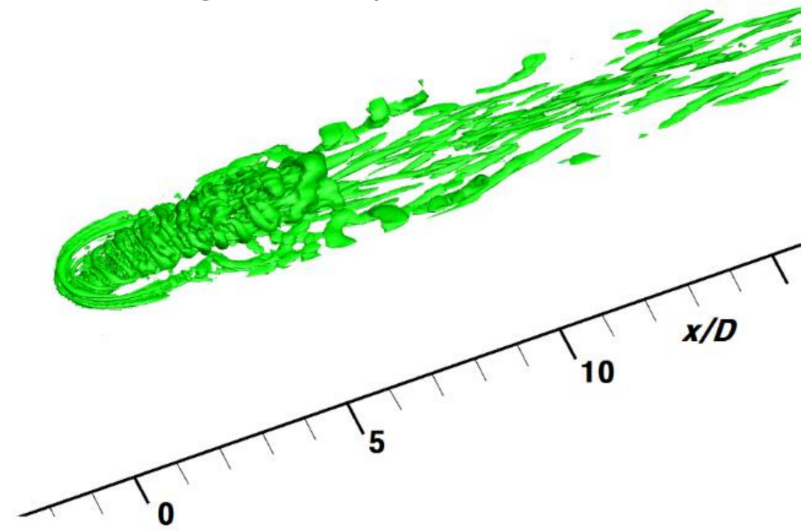

(c) Single-hole system at $32 \mathrm{~Hz}$

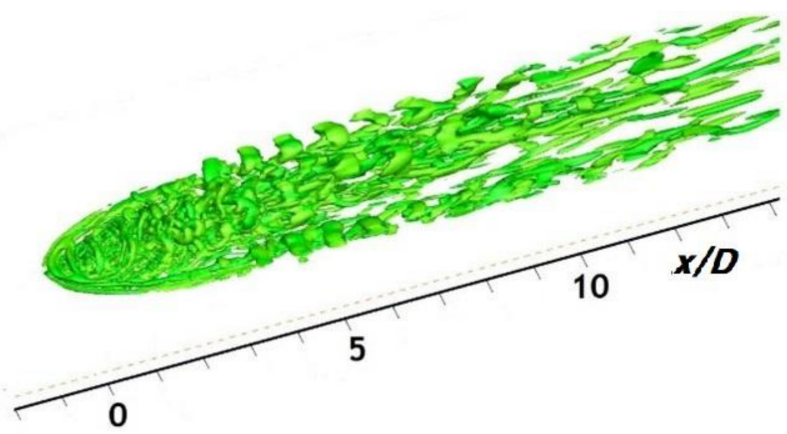

(b) Triple-hole system at $0 \mathrm{~Hz}[3]$

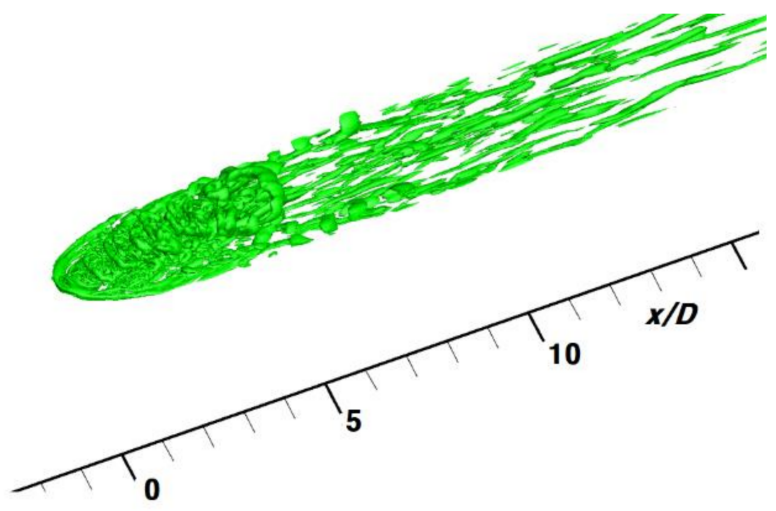

(d) Triple-hole system at $32 \mathrm{~Hz}$

Figure 18. Q-criterion contours (level: 80,000).

The instantaneous Q-contours at $32 \mathrm{~Hz}$ were captured when the coolant velocity at the exit was higher than its average value, and therefore, it illustrated various vortex structures like the kidney vortex, Kelvin-Helmholtz vortices, hairpin and horseshoe vortices more clearly than at $0 \mathrm{~Hz}$. However, Q-criterion contours for the triple-hole configuration at 0 and $32 \mathrm{~Hz}$ showed smaller hairpin and Kelvin-Helmholtz vortices than those for the single-hole configuration. These smaller hairpin vortices were related to weakened Counter Rotating Vortex Pair [35]. A weakened Counter Rotating Vortex Pair increased $\eta$ on the wall.

\section{Conclusions}

The mainstream of film cooling can be disturbed by several factors. The specific goal of the current study is to investigate the effects of the main flow pulsation on the film cooling performance for the triple holes system in order to help for making better cooling design of the gas turbine engines. Film cooling in a triple hole system was numerically simulated in an LES. Contours of $\eta$ on the wall and cross-sectional views of the mean dimensionless temperature contours for the single-hole and triple-hole systems showed that the triple-hole configuration exhibits better film cooling performance for $0,2,16$, and $32 \mathrm{~Hz}$ pulsations. In the triple-hole system, increasing the frequency to $32 \mathrm{~Hz}$ or Strouhal number from 0 to 1.6085 decreased the film cooling effectiveness to a lesser extent compared with the single-hole system. As the frequency increased from 0 to 2, 16, and $32 \mathrm{~Hz}$ in the single-hole, laterally averaged $\eta$ decreased by approximately $11 \%, 12 \%$, and $45 \%$, respectively, and this trend was confirmed in the experimental data. In the triple hole, increasing the frequency 
from 0 to 2, 16, and $32 \mathrm{~Hz}$ decreased the spanwise-averaged film cooling effectiveness by approximately $4 \%, 5 \%$, and $39 \%$, respectively. The film cooling effectiveness was always higher in the triple-hole system because of the generation of an anti-CRVP. Furthermore, increasing the pulsation frequency or Strouhal number increased the ratio of $\eta_{m \text {, triple hole }}$ to $\eta_{m \text {, single hole }}$, indicating that at higher pulsation frequencies or Strouhal numbers in the main flow, the triple holes were more effective for film cooling compared with the single hole. Contours of time-averaged velocity magnitude in sister holes showed that the flow was more developed than that in the main hole, even at $32 \mathrm{~Hz}$ pulsation. This indicated that the sister holes improved the film cooling effectiveness through the generation of an anti-CRVP and by increasing the $L / D$ ratios. Q-contours for triple hole at 0 and $32 \mathrm{~Hz}$ showed smaller hairpin and jet shear layer vortices compared with those for the single-hole system, indicating a weakened CRVP. In the contours of the instantaneous film cooling effectiveness, the horseshoe vortex was visible periodically at $32 \mathrm{~Hz}$ pulsation because this vortex was generated when the vortex in the boundary layer of the main flow was blocked by strong coolant injection and roll-up. This paper only covered the cylindrical hole, however, the effects of the main flow pulsations on film cooling for the shaped hole such as the forward expansion hole will be covered in future studies. Moreover, the effects for the two sister holes positioned more downstream of the primary hole and the optimized length between the sister hole and the primary hole will be investigated to obtain the best film cooling performance. For the parametric study, the CFD results of this study could be used as the baseline.

Author Contributions: Simulations, S.I.B.; analysis, S.I.B. and J.A.; writing, S.I.B. and J.A.; supervision, J.A. All authors have read and agreed to the published version of the manuscript.

Funding: No external funding.

Institutional Review Board Statement: Not applicable.

Informed Consent Statement: Not applicable.

Data Availability Statement: Data is contained within this article.

Conflicts of Interest: No conflict of interest.

\section{Abbreviations}

$\begin{array}{ll}D & \text { diameter of single hole } \\ L & \text { hole length } \\ M & \text { blowing ratio }=\left(\rho_{C} U_{C}\right) /\left(\rho_{G} U_{G}\right) \\ \mathrm{Sr} & \text { Strouhal number }=\frac{2 \pi f L}{U_{G}} \\ U & \text { flow velocity } \\ \text { Greek symbols } & \\ \eta & \text { film cooling effectiveness } \\ \eta_{C} & \text { effectiveness at the centerline } \\ \eta_{m} & \text { laterally-averaged effectiveness } \\ \Theta & \text { dimensionless temperature }=\frac{\left(T_{G}-T\right)}{T_{G}-T_{C}}\end{array}$

\section{References}

1. Moran, M.; Shapiro, H.; Boettner, D.; Bailey, M. Fundamentals of Engineering Thermodynamics, 8th ed.; John Wiley \& Sons: Hoboken, NJ, USA, 2014; p. 532.

2. Baek, S.I.; Yavuzkurt, S. Effects of Flow Oscillations in the Mainstream on Film Cooling. Inventions 2018, 3, 73. [CrossRef]

3. Baek, S.I.; Ahn, J. Large Eddy Simulation of Film Cooling with Triple Holes: Injectant Behavior and Adiabatic Film-Cooling Effectiveness. Processes 2020, 8, 1443. [CrossRef]

4. Heidmann, J.; Ekkad, S. A novel anti-vortex turbine film cooling hole concept. In Proceedings of the ASME Turbo Expo 2007, GT2007-27528, Montreal, QC, Canada, 14-17 May 2007. [CrossRef]

5. Javadi, K.; Taeibi-Rahni, M.; Darbandi, M.; Javadi, K. Jet-into-Crossflow Boundary-Layer Control: Innovation in Gas Turbine Blade Cooling. AIAA J. 2007, 45, 2910-2925. [CrossRef] 
6. Ely, M.J.; Jubran, B.A. A numerical evaluation on the effect of sister holes on film cooling effectiveness and the surrounding flow field. Heat Mass Transf. 2009, 45, 1435-1446. [CrossRef]

7. Choi, D.-W.; Lee, K.-D.; Kim, K.-Y. Analysis and Optimization of Double-Jet Film-Cooling Holes. J. Thermophys. Heat Transf. 2013, 27, 246-254. [CrossRef]

8. Wu, H.; Cheng, H.; Li, Y.; Rong, C.; Ding, S. Effects of side hole position and blowing ratio on sister hole film cooling performance in a flat plate. Appl. Therm. Eng. 2016, 93, 718-730. [CrossRef]

9. Seo, H.; Leea, J.; Ligrani, P. The effect of injection hole length on film cooling with bulk flow pulsations. Int. J. Heat Mass Transf. 1998, 41, 3515-3528. [CrossRef]

10. Kim, S.I.; Hassan, I. Unsteady Simulations of a Film Cooling Flow from an Inclined Cylindrical Jet. J. Thermophys. Heat Transf. 2010, 24, 145-156. [CrossRef]

11. Gau, C.; Yih, K.A.; Chang, S. Swirling flow effect on film cooling performance downstream of a sudden expansion. J. Thermophys. Heat Transf. 1991, 5, 89-95. [CrossRef]

12. Coulthard, S.M.; Volino, R.J.; Flack, K.A. Effect of Jet Pulsing on Film Cooling-Part I: Effectiveness and Flow-Field Temperature Results. J. Turbomach. 2006, 129, 232-246. [CrossRef]

13. Nikitopoulos, D.E.; Acharya, S.; Oertling, J.; Muldoon, F.H. On Active Control of Film-Cooling Flows. In Proceedings of the ASME Turbo Expo, GT2006-90051, Barcelona, Spain, 8-11 May 2006. [CrossRef]

14. Ekkad, S.V.; Ou, S.; Rivir, R.B. Effect of Jet Pulsation and Duty Cycle on Film Cooling from a Single Jet on a Leading Edge Model. J. Turbomach. 2006, 128, 564-571. [CrossRef]

15. Gao, Z.; Narzary, D.P.; Mhetras, S.; Han, J. Upstream Vortex Effects on Turbine Blade Platform Film Cooling with Typical Purge Flow. J. Thermophys. Heat Transf. 2012, 26, 75-84. [CrossRef]

16. Xiao, B.; Wang, W.; Zhang, X.; Long, G.; Fan, J.; Chen, H.; Deng, L. A novel fractal solution for permeability and Kozeny-Carman constant of fibrous porous media made up of solid particles and porous fibers. Powder Technol. 2019, 349, 92-98. [CrossRef]

17. Liang, M.; Fu, C.; Xiao, B.; Luo, L.; Wang, Z. A fractal study for the effective electrolyte diffusion through charged porous media. Int. J. Heat Mass Transf. 2019, 137, 365-371. [CrossRef]

18. Farhadi-Azar, R.; Ramezanizadeh, M.; Taeibi-Rahni, M.; Salimi, M. Compound Triple Jets Film Cooling Improvements via Velocity and Density Ratios: Large Eddy Simulation. J. Fluids Eng. 2011, 133, 031202. [CrossRef]

19. Johnson, P.L.; Kapat, J.S. Large-Eddy Simulations of a Cylindrical Film Cooling Hole. J. Thermophys. Heat Transf. 2013, 27, 255-273. [CrossRef]

20. Baldauf, S.; Scheurlen, M.; Schulz, A.; Wittig, S. Correlation of Film-Cooling Effectiveness from Thermographic Measurements at Enginelike Conditions. J. Turbomach. 2002, 124, 686-698. [CrossRef]

21. Babaee, H.; Wan, X.; Acharya, S. Effect of Uncertainty in Blowing Ratio on Film Cooling Effectiveness. J. Heat Transf. 2013, 136, 031701. [CrossRef]

22. Bogard, D. Airfoil Film Cooling. In The Gas Turbine Handbook; National Energy Technology Laboratory: Albany, NY, USA, 2006.

23. Seo, H. Film Cooling Flow and Heat Transfer Characteristics with the Strouhal Number Variations in Pulsating Flows. Ph.D. Thesis, Seoul National University, Seoul, Korea, 1997.

24. ANSYS Fluent Theory Guide. Version 19. Available online: https://www.ansys.com/products/fluids/ansys-fluent (accessed on 10 December 2020).

25. Pointwise Version 18. Available online: http:/ / www.pointwise.com/ (accessed on 10 December 2020).

26. Renze, P.C.-A.; Schröder, W.; Meinke, M. Large-eddy Simulation of Film Cooling Flows with Variable Density Jets. Flow Turbul. Combust. 2007, 80, 119-132. [CrossRef]

27. Iourokina, I.; Lele, S. Towards large eddy simulation of film cooling flows on a model turbine blade leading edge. In Proceedings of the 43rd AIAA Aerospace Sciences Meeting and Exhibit, No. 2005-0670, Reno, NV, USA, 10-13 January 2005.

28. Acharya, S.; Leedom, D.H. Large Eddy Simulations of Discrete Hole Film Cooling with Plenum Inflow Orientation Effects. J. Heat Transf. 2012, 135, 011010. [CrossRef]

29. White, F. Fluid Mechanics, 4th ed.; McGraw-Hill: New York, NY, USA, 1999.

30. Jung, I.S.; Lee, J.S. Effects of Orientation Angles on Film Cooling Over a Flat Plate: Boundary Layer Temperature Distributions and Adiabatic Film Cooling Effectiveness. J. Turbomach. 1999, 122, 153-160. [CrossRef]

31. Fric, T.F.; Roshko, A. Vortical structure in the wake of a transverse jet. J. Fluid Mech. 1994, 279, 1-47. [CrossRef]

32. Shyam, V.; Thurman, D.; Poinsatte, P.; Ameri, A.A.; Eichele, P. Long Hole Film Cooling Dataset for CFD Development-Flow and Film Effectiveness. In Proceedings of the 50th AIAA/ASME/SAE/ASEE Joint Propulsion Conference, Cleveland, OH, USA, 28-30 July 2014; American Institute of Aeronautics and Astronautics (AIAA): Reston, VA, USA, 2014.

33. Kolář, V. Vortex identification: New requirements and limitations. Int. J. Heat Fluid Flow 2007, 28, 638-652. [CrossRef]

34. Schroder, A.; Willert, C. Particle Image Velocimetry: New Developments and Recent Applications; Springer: Berlin/Heidelberg, Germany, 2008; p. 382.

35. Yao, Y.; Zhang, J.; Yang, Y. Numerical study on film cooling mechanism and characteristics of cylindrical holes with branched jet injections. Propuls. Power Res. 2013, 2, 30-37. [CrossRef] 